\title{
ON THE $\Pi_{\gamma}^{0}$-COMPLETENESS AND $\Sigma_{\gamma}^{0}$-COMPLETENESS OF MULTIFRACTAL DECOMPOSITION SETS
}

\author{
L. OLSEN \\ Department of Mathematics \\ University of St. Andrews \\ St. Andrews, Fife KY16 9SS, Scotland \\ e-mail: lo@st-and.ac.uk
}

\begin{abstract}
The purpose of this paper twofold. Firstly, we establish $\Pi_{\gamma}^{0}$-completeness and $\Sigma_{\gamma}^{0}$-completeness of several different classes of multifractal decomposition sets of arbitrary Borel measures (satisfying a mild non-degeneracy condition and two mild "smoothness" conditions). Secondly, we apply these results to study the $\Pi_{\gamma}^{0}$-completeness and $\Sigma_{\gamma}^{0}$-completeness of several multifractal decomposition sets of self-similar measures (satisfying a mild separation condition). For example, a corollary of our results shows if $\mu$ is a self-similar sets satisfying the Strong Separation Condition and $\mu$ is not equal to the normalised Hausdorff measure on
\end{abstract} its support, then the classical multifractal decomposition sets of $\mu$ defined by

$$
\left\{x \in \mathbb{R}^{d} \mid \lim _{r \searrow 0} \frac{\log \mu(B(x, r))}{\log r}=\alpha\right\}
$$

are $\Pi_{3}^{0}$-complete provided they are non-empty.

\section{INTRODUCTION}

Ki \& Linton [KiLi] have recently in a remarkable paper determined the exact location of the set of normal numbers in the Borel Hierarchy. The purpose of this paper twofold. Firstly, we show that the methods and techniques from Ki \& Linton's paper [KiLi] can be used to establish $\Pi_{\gamma}^{0}$-completeness and $\Sigma_{\gamma}^{0}$-completeness of several different classes of multifractal decomposition sets of arbitrary Borel measures (satisfying a mild non-degeneracy condition and two mild "smoothness" conditions). Secondly, we apply these results to study the $\Pi_{\gamma}^{0}$-completeness and $\Sigma_{\gamma}^{0}$-completeness of several multifractal decomposition sets of a special class of important measures occurring often and naturally in fractal geometry and dynamical systems, namely, the class of self-similar measures (satisfying a mild separation condition).

In Section 1.1 we recall the Borel Hierarchy and in Section 1.2 we introduce $\Pi_{\gamma}^{0}$-completeness and $\Sigma_{\gamma}^{0}$-completeness. In Section 1.3 we recall basic notions from multifractal analysis and introduce the fundamental multifractal decomposition sets that we will analyse. Finally, in Section 2 and Section 3 we present our main results; Section 2 presents our results on arbitrary measures and in Section 3 we present our results on self-similar measures. The proofs are given in Sections 4-7.

1.1. The Borel Hierarchy. We will now briefly describe the Borel Hierarchy . Let $X$ be a metric space. For an ordinal $\gamma$ with $1 \leq \gamma<\omega_{1}$ (where $\omega_{1}$ is the first uncountable cardinal), the Baire classes $\Sigma_{\gamma}^{0}(X)=\Sigma_{\gamma}^{0}$ and $\Pi_{\gamma}^{0}(X)=\Pi_{\gamma}^{0}$ are defined inductively by

$$
\Sigma_{1}^{0}(X)=\{G \subseteq X \mid G \text { is open }\}, \quad \Pi_{1}^{0}(X)=\{F \subseteq X \mid F \text { is closed }\}
$$

and

$$
\Sigma_{\gamma}^{0}(X)=\left\{\bigcup_{n=1}^{\infty} E_{n} \mid E_{n} \in \bigcup_{\kappa<\gamma} \Pi_{\kappa}^{0}(X)\right\}, \quad \Pi_{\gamma}^{0}(X)=\left\{\bigcap_{n=1}^{\infty} E_{n} \mid E_{n} \in \bigcup_{\kappa<\gamma} \Sigma_{\kappa}^{0}(X)\right\} .
$$

2000 Mathematics Subject Classification. 28A80, 03E15, 54A05.

Key words and phrases: Multifractals, self-similar measures, descriptive set theory, $\Pi_{\gamma}^{0}$-completeness, $\Sigma_{\gamma}^{0}$-completeness 
We then have the following diagram

$$
\begin{array}{llll}
\Sigma_{1}^{0}(X) & \Sigma_{2}^{0}(X) & \Sigma_{3}^{0}(X) & \ldots \\
\Pi_{1}^{0}(X) & \Pi_{2}^{0}(X) & \Pi_{3}^{0}(X) & \ldots
\end{array}
$$

in which any Baire class is contained in any Baire class to the right of it; this is known as the Borel Hierarchy, cf. [Ke, p. 68]. Is is known that $\cup_{\gamma<\omega_{1}} \Sigma_{\gamma}^{0}=\cup_{\gamma<\omega_{1}} \Pi_{\gamma}^{0}=\mathcal{B}(X)$ where $\mathcal{B}(X)$ denotes the Borel $\sigma$-algebra on $X$. The Borel Hierarchy gives a ramafication of the Borel sets in (at least) $\omega_{1}$ levels. Sometimes we will use the traditional notation, $\mathcal{G}(X)=\mathcal{G}$, for the family of open subsets of $X$, and the traditional notation, $\mathcal{F}(X)=\mathcal{F}$, for the family of closed subsets of $X$. Hence, $\Sigma_{1}^{0}(X)=\mathcal{G}, \Pi_{1}^{0}(X)=\mathcal{F}$, $\Sigma_{2}^{0}(X)=\mathcal{F}_{\sigma}, \Pi_{2}^{0}(X)=\mathcal{G}_{\delta}$, and so on.

1.2. Reducibility, hardness and completeness. The following terminology is useful for discussing the exact position of sets in the Borel hierarchy.

Reducibility. Let $X$ and $Y$ be a metric space and let $A \subseteq X$ and $P, Q \subseteq Y$. We will say that $A$ is (Wadge) reducible to $P$ and $Q$ if there is continuous map $f: X \rightarrow Y$ such that

$$
f(A) \subseteq P, \quad f(X \backslash A) \subseteq Q
$$

If $A$ is reducible to $P$ and $Q$, then we will write

$$
(X ; A) \underset{\mathrm{w}}{\leq}(Y ; P, Q)
$$

Also, if $A$ is reducible to $P$ and $Y \backslash P$, then we will write

$$
(X ; A) \underset{\mathrm{w}}{\leq}(Y ; P)
$$

We can now define (Wadge) hardness and (Wadge) completeness.

Definition. Hardness. Let $X$ be a metric space and let $\gamma$ be an ordinal with $\gamma<\omega_{1}$. A subset $A$ of $X$ is called $\Pi_{\gamma}(X)$-hard if for all $M \in \Pi_{\gamma}\left(\{1,2\}^{\mathbb{N}}\right)$, we have

$$
\left(\{1,2\}^{\mathbb{N}} ; M\right) \leq \underset{\mathrm{w}}{\leq}(X ; A) .
$$

Similarly, a subset $E$ of $X$ is called $\Sigma_{\gamma}(X)$-hard if for all $M \in \Sigma_{\gamma}\left(\{1,2\}^{\mathbb{N}}\right)$, we have

$$
\left(\{1,2\}^{\mathbb{N}} ; M\right) \leq \underset{\mathrm{w}}{ }(X ; A) .
$$

Definition. Completeness. Let $X$ be a metric space and let $\gamma$ be an ordinal with $\gamma<\omega_{1}$. A subset $E$ of $X$ is called $\Pi_{\gamma}(X)$-complete if $E$ is $\Pi_{\gamma}(X)$-hard and $E \in \Pi_{\gamma}(X)$. Similarly, a subset $E$ of $X$ is called $\Sigma_{\gamma}(X)$-complete if $E$ is $\Sigma_{\gamma}(X)$-hard and $E \in \Sigma_{\gamma}(X)$

1.3. Multifractal decomposition sets of measures. During the past 20 years the multifractal structure of measures has received much attention in the literature. Let $\lambda$ be a Borel measure on $\mathbb{R}^{d}$. Multifractal analysis refers to the study of the fractal geometry of the sets of those points $x$ for which the measure $\lambda(B(x, r))$ of the ball $B(x, r)$ with center $x$ and radius $r$ behaves like $r^{\alpha}$ for small $r$, i.e. the set

$$
\Delta_{\lambda}(\alpha)=\left\{\begin{array}{l|l}
x \in \mathbb{R}^{d} & \lim _{r \searrow 0} \frac{\log \lambda(B(x, r))}{\log r}=\alpha
\end{array}\right\}
$$

If the intensity of the measure $\lambda$ varies very widely, it may happen that the sets $\Delta_{\lambda}(\alpha)$ display a fractallike character for a range of values of $\alpha$. If this is the case, then the measure is called a multifractal measure or simply a multifractal, and it is natural to study the sizes of the sets $\Delta_{\lambda}(\alpha)$ as $\alpha$ varies. 
In particular, it is natural to study the Hausdorff dimension of the sets $\Delta_{\lambda}(\alpha)$ as a function of $\alpha$. Motivated by this, we define the multifractal spectrum $f_{\lambda}$ of $\lambda$ by

$$
\begin{aligned}
& f_{\lambda}(\alpha)=\operatorname{dim}_{H} \Delta_{\lambda}(\alpha)
\end{aligned}
$$

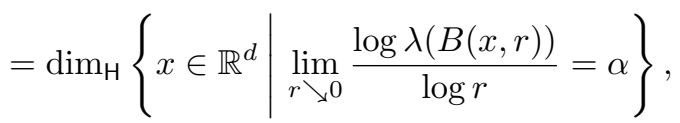

for $\alpha \geq 0$, where $\operatorname{dim}_{H}$ denotes the Hausdorff dimension. The function in (1.2) is known as the multifractal spectrum of $\mu$, and one of the main problems in multifractal analysis is to study this and related functions, see, for example, $[\mathrm{Fa}, \mathrm{Pe}]$ and the references therein. For $A \subseteq \mathbb{R}$, it is also natural to investigate the more general multifractal decomposition set defined by

$$
\Delta_{\lambda}(A)=\left\{x \in \mathbb{R}^{d} \mid \lim _{r \searrow 0} \frac{\log \lambda(B(x, r))}{\log r} \in A\right\} .
$$

Note that if $A=\{\alpha\}$ for $\alpha \in \mathbb{R}$, then

$$
\Delta_{\lambda}(\{\alpha\})=\Delta_{\lambda}(\alpha) .
$$

We also define the set of admissible values of $\lambda$ by

$$
I_{\lambda}=\left\{\alpha \in \mathbb{R} \mid \Delta_{\lambda}(\alpha) \neq \varnothing\right\}
$$

The purpose of this paper is to analyse the set-theoretical complexity of the multifractal decomposition sets $\Delta_{\lambda}(A)$ and related sets. In particular, we determine their exact location in the Borel hierarch and we establish $\Pi_{\gamma}^{0}$-completeness and $\Sigma_{\gamma}^{0}$-completeness of the sets. This is done in Section 2 and Section 3 below. In Section 2 we present our main results for arbitrary measures, and in Section 3 we apply the results from Section 2 to study a special class of important measures occurring often and naturally in fractal geometry and dynamical systems, namely, the class of self-similar measures (satisfying a mild separation condition).

\section{Results for Arbitrary Measures}

2.1. Upper bounds for arbitrary measures. In this section we presents "upper bounds" for the position of the multifractal decomposition set $\Delta_{\lambda}(A)$ in the Borel Hierarchy. In order to obtain meaningful results we must impose a non-degeneracy on the measure $\lambda$ ensuring that $\lambda$ is not supported on lower dimensional sub-manifolds of $\mathbb{R}^{d}$; this condition is presented in the definition below.

Definition. Aspherical measure. For $x \in \mathbb{R}^{d}$ and $r>0$, we define the sphere with centre at $x$ and radius $r$ by $S(x, r)=\left\{y \in \mathbb{R}^{d}|| x-y \mid=r\right\}$. A Borel measure $\lambda$ on $\mathbb{R}^{d}$ is called aspherical if

$$
\lambda(S(x, r))=0
$$

for all $x \in \mathbb{R}^{d}$ and all $r>0$.

We note that many natural and important classes of measures are aspherical; for example, self similar measures satisfying the Strong Separation Condition are aspherical by Lemma 3.1.

We can now provide "upper bounds" for the position of the multifractal decomposition set $\Delta_{\lambda}(A)$ in the Borel Hierarchy. More precisely, we prove that if $\gamma$ is finite, then the position of $\Delta_{\lambda}(A)$ in the Borel Hierarchy is at most one level further to the left of the position of $A$, and if $\gamma$ is infinite, then the position of $\Delta_{\lambda}(A)$ in the Borel Hierarchy is at the same level as the position of $A$; this is the contents of the next theorem.

Theorem 2.1. Upper bounds for arbitrary measures. Let $\lambda$ be a Borel measure on $\mathbb{R}^{d}$ and assume that $\lambda$ is aspherical. Let $\gamma$ be an ordinal with $\gamma<\omega_{1}$.

(1) If $A \in \Pi_{\gamma}^{0}$, then $\Delta_{\lambda}(A) \in \begin{cases}\Pi_{\gamma+1}^{0} & \text { for } 2 \leq \gamma<\omega ; \\ \Pi_{\gamma}^{0} & \text { for } \omega \leq \gamma .\end{cases}$ 
(2) If $A \in \Sigma_{\gamma}^{0}$, then $\Delta_{\lambda}(A) \in \begin{cases}\Sigma_{\gamma+1}^{0} & \text { for } 3 \leq \gamma<\omega ; \\ \Sigma_{\gamma}^{0} & \text { for } \omega \leq \gamma .\end{cases}$

The proof of Theorem 2.1 are given in Sections 4-5, and is based on transfinite inductive.

2.2. Lower bounds for arbitrary measures. We will now obtain "lower bounds" for the position of the multifractal decomposition set $\Delta_{\lambda}(A)$ in the Borel Hierarchy. In particular, we will establish the $\Pi_{\gamma}^{0}$-completeness $/ \Sigma_{\gamma}^{0}$-completeness of the sets $\Delta_{\lambda}(A)$ leading to an exact classification of the position of the multifractal decomposition sets $\Delta_{\lambda}(A)$ in the Borel Hierarchy. Lower bounds and $\Pi_{\gamma^{-}}^{0}$ completeness $/ \Sigma_{\gamma}^{0}$-completeness are established by showing that the sets $\Delta_{\lambda}(A)$ are reducible to sets that are already known to be $\Pi_{\gamma}^{0}$-complete $/ \Sigma_{\gamma}^{0}$-complete. However, in order to construct continuous maps establishing the reducibility between a known $\Pi_{\gamma}^{0}$-complete $/ \Sigma_{\gamma}^{0}$-complete set and $\Delta_{\lambda}(A)$, some degree of "smoothness" is needed. In particular, we impose the following two mild "smoothness" conditions. The first condition (the existence of a continuous selector) specifies the existence of a continuous choice function from the set of admissible values $I_{\lambda}$ into the union $\cup_{\alpha \in I_{\lambda}} \Delta_{\lambda}(\alpha)$; this condition allow us to construct continuous functions while proving $\Pi_{\gamma}^{0}$-completeness $/ \Sigma_{\gamma}^{0}$-completeness of $\Delta_{\lambda}(\alpha)$. The second condition (the admissible condition) implies the existence a complete zero-dimensional metric space, namely, the Baire space $\mathbb{N}^{\mathbb{N}}$, that is homeomorphic to a dense subset of the set $I_{\lambda}$ of admissible values; this "smoothness" condition allows us to reduce questions of $\Pi_{\gamma}^{0}$-completeness $/ \Sigma_{\gamma}^{0}$-completeness from $\mathbb{R}^{d}$ to the Baire space $\mathbb{N}^{\mathbb{N}}$ making it significantly less difficult to establish $\Pi_{\gamma}^{0}$-completeness $/ \Sigma_{\gamma}^{0}$-completeness (because a subset of a complete zero-dimensional metric space is $\Pi_{\gamma}^{0}$-complete $\left(\Sigma_{\gamma}^{0}\right.$-complete) if and only it is in $\Pi_{\gamma}^{0} \backslash \Sigma_{\gamma}^{0}\left(\Sigma_{\gamma}^{0} \backslash \Pi_{\gamma}^{0}\right)$, see, for example, [Ke, Theorem 22.10]). We now state the required "smoothness" conditions. Recall, that the set $I_{\lambda}$ of admissible values of $\lambda$ is defined in (1.4).

Definition. Measure with continuous selector. A Borel measure $\lambda$ on $\mathbb{R}^{d}$ is said to have a continuous selector if there is continuous function $\mathcal{S}: I_{\lambda} \rightarrow \mathbb{R}^{d}$ such that

$$
\mathcal{S}(\alpha) \in \Delta_{\lambda}(\alpha)
$$

for all $\alpha \in I_{\lambda}$.

Definition. Admissible measure. A Borel measure $\lambda$ on $\mathbb{R}^{d}$ is said to be admissible if there is a subset $U$ of $I_{\lambda}$ such that:

(1) The set $I_{\lambda} \backslash U$ is countable.

(2) The sets $[0,1] \cap \mathbb{I}$ and $U$ are homeomorphic; here $\mathbb{I}$ denotes the set of irrationals.

Again, we note that many natural and important classes of measures have continuous selectors and are admissible; for example, self similar measures satisfying the Strong Separation Condition have continuous selectors and are admissible by Lemma 3.1.

We can now state our second and third main results establishing the $\Pi_{\gamma}^{0}$-hardness $/ \Sigma_{\gamma}^{0}$-hardness and $\Pi_{\gamma}^{0}$-completeness $/ \Sigma_{\gamma}^{0}$-completeness of the sets $\Delta_{\lambda}(A)$ leading to an exact classification of the position of the multifractal decomposition sets $\Delta_{\lambda}(A)$ in the Borel Hierarchy.

Theorem 2.2. Lower bounds for arbitrary measures: hardness. Let $\lambda$ be a Borel measure on $\mathbb{R}^{d}$ and assume that $\lambda$ has a continuous selector and that $\lambda$ is admissible. Let $\gamma$ be an ordinal with $\gamma<\omega_{1}$.

(1) If $A \subseteq I_{\lambda}$ is $\Pi_{\gamma}^{0}$-hard, then $\Delta_{\lambda}(A)$ is $\Pi_{\gamma}^{0}$-hard for $\gamma \geq 3$.

(2) If $A \subseteq I_{\lambda}$ is $\Sigma_{\gamma}^{0}$-hard, then $\Delta_{\lambda}(A)$ is $\Sigma_{\gamma}^{0}$-hard for $\gamma \geq 3$. 
Theorem 2.3. Lower bounds for arbitrary measures: completeness. Let $\lambda$ be a Borel measure on $\mathbb{R}^{d}$ and assume that $\lambda$ is aspherical, that $\lambda$ has a continuous selector, and that $\lambda$ is admissible. Let $\gamma$ be an ordinal with $\gamma<\omega_{1}$.

(1) If $A \subseteq I_{\lambda}$ is $\Pi_{\gamma}^{0}\left(I_{\lambda}\right)$-complete, then $\Delta_{\lambda}(A)$ is $\begin{cases}\Pi_{\gamma+1}^{0} \text {-complete } & \text { for } 3 \leq \gamma<\omega \text {; } \\ \Pi_{\gamma}^{0} \text {-complete } & \text { for } \omega \leq \gamma .\end{cases}$

(2) If $A \subseteq I_{\lambda}$ is $\Sigma_{\gamma}^{0}\left(I_{\lambda}\right)$-complete, then $\Delta_{\lambda}(A)$ is $\begin{cases}\Sigma_{\gamma+1}^{0} \text {-complete } & \text { for } 3 \leq \gamma<\omega \text {; } \\ \Sigma_{\gamma}^{0} \text {-complete } & \text { for } \omega \leq \gamma .\end{cases}$

The proofs of Theorems 2.2-2.3 are given in Section 6 .

\section{Results for Self-Similar Measures}

We will now apply the results in Section 2 to provide a more detailed analysis of the set-theoretical complexity of the multifractal decomposition sets $\Delta_{\mu}(A)=\left\{x \in \mathbb{R}^{d} \mid \lim _{r \searrow 0} \frac{\log \mu(B(x, r))}{\log r} \in A\right\}$ and related sets for self-similar measures $\mu$.

3.1. Self-similar measures. Let $S_{i}: \mathbb{R}^{d} \rightarrow \mathbb{R}^{d}$ for $i=1, \ldots, N$ be contracting similarities and let $\left(p_{1}, \ldots, p_{N}\right)$ be a probability vector. For each $i$, we denote the Lipschitz constant of $S_{i}$ by $r_{i} \in(0,1)$. Let $K$ and $\mu$ be the self-similar set and the self-similar measure associated with the list $\left(S_{1}, \ldots, S_{N}, p_{1}, \ldots, p_{N}\right)$, i.e. $K$ is the unique non-empty compact subset of $\mathbb{R}^{d}$ such that

$$
K=\bigcup_{i} S_{i}(K),
$$

and $\mu$ is the unique Borel probability measure on $\mathbb{R}^{d}$ such that

$$
\mu=\sum_{i} p_{i} \mu \circ S_{i}^{-1}
$$

cf. $[\mathrm{Hu}]$.

3.2. Multifractal decomposition sets of self-similar measures. During the past 20 years the multifractal spectrum of the self-similar measure $\mu$ in (3.2) has received much attention in the literature. Assuming various so-called separation conditions, i.e. conditions guaranteeing that the overlaps between the sets $S_{i}(K)$ and $S_{j}(K)$ for $i \neq j$ are small, many authors have investigated the multifractal spectrum $f_{\mu}(\alpha)$. A commonly used separation condition is the Strong Separation Condition (SSC). The SSC is defined as follows.

Definition. The SSC. The list $\left(S_{1}, \ldots, S_{N}\right)$ is said to satisfy the $S S C$ provided $S_{i}(K) \cap S_{j}(K)=\varnothing$ for all $i, j=1, \ldots, N$ with $i \neq j$ (where $K$ is the self-similar set in (3.1)).

For example, the multifractal spectrum of a self-similar measure (and more general measures) satisfying the SSC (and significantly weaker separation conditions) have been computed within the past 10-15 years. The reader is referred to the textbooks $[\mathrm{Fa}, \mathrm{Pe}]$ for a more detailed discussion these problems. It is useful to introduce following notation, namely, let

$$
\alpha_{\min }=\min _{i} \frac{\log p_{i}}{\log r_{i}}, \quad \alpha_{\max }=\max _{i} \frac{\log p_{i}}{\log r_{i}} .
$$

and observe that it is well-known that if $\mu$ satisfies the SSC, then

$$
I_{\mu}=\left[\alpha_{\min }, \alpha_{\max }\right],
$$


see, for example, $[\mathrm{Fa}]$.

We note the following. Let $K$ and $\mu$ be the self-similar set and self-similar measure in (3.1) and (3.2), respectively, and assume that the SSC is satisfied. If $\left(p_{1}, \ldots, p_{N}\right)=\left(r_{1}^{s}, \ldots, r_{N}^{s}\right)$ where $s=\operatorname{dim}_{\mathrm{H}}(K)$, then it is known that $\Delta_{\mu}(A)$ is compact or empty, indeed, we have

$$
\Delta_{\mu}(A)= \begin{cases}K & \text { if } s \in A ; \\ \varnothing & \text { if } s \notin A ;\end{cases}
$$

see, for example, $[\mathrm{Fa}]$ or $[\mathrm{Pe}]$. Hence, if $\left(p_{1}, \ldots, p_{N}\right)=\left(r_{1}^{s}, \ldots, r_{N}^{s}\right)$, then the set theoretical structure of $\Delta_{\mu}(A)$ is well understood, and below we will therefore only consider the case for which $\left(p_{1}, \ldots, p_{N}\right) \neq$ $\left(r_{1}^{s}, \ldots, r_{N}^{s}\right)$.

We also note that it follows from (3.4) that

$$
\Delta_{\mu}(A)=\Delta_{\mu}\left(A \cap\left[\alpha_{\min }, \alpha_{\max }\right]\right) .
$$

Because of this we can assume that $A \subseteq\left[\alpha_{\min }, \alpha_{\max }\right]$ below.

Finally, we note that self-similar measures satisfying the SSC are aspherical, have continuous selectors, and are admissible; this is the contents of the next lemma.

Lemma 3.1. Let $\mu$ be the self-similar measure in (3.2) and assume that the SSC is satisfied. Then the measure $\mu$ is aspherical, the measure $\mu$ has a continuous selector, and the measure $\mu$ is admissible.

The proof of Lemma 3.1 is given in Section 7.1

Next, we turn towards the discussion of the $\Pi_{\gamma}^{0}$-hardness $/ \Sigma_{\gamma}^{0}$-hardness and $\Pi_{\gamma}^{0}$-completeness $/ \Sigma_{\gamma}^{0}$ completeness of the multifractal decomposition sets $\Delta_{\mu}(A)$ of self-similar measures $\mu$.

We first discuss hardness of $\Delta_{\mu}(A)$ for the self-similar measure $\mu$ in (3.2). Indeed, it follows from Lemma 3.1, that we can apply Theorem 2.2 to the self-similar measure $\mu$ in (3.2), and we therefore immediately conclude from Theorem 2.2 that if $A \subseteq\left[\alpha_{\min }, \alpha_{\max }\right]$ is $\Pi_{\gamma}^{0}\left(\left[\alpha_{\min }, \alpha_{\max }\right]\right)$-hard, then

$$
\left\{x \in \mathbb{R}^{d} \mid \lim _{r \searrow 0} \frac{\log \mu(B(x, r))}{\log r} \in A\right\} \text { is } \quad \Pi_{\gamma}^{0} \text {-hard } \quad \text { for } 3 \geq \gamma
$$

and if $A \subseteq\left[\alpha_{\min }, \alpha_{\max }\right]$ is $\Sigma_{\gamma}^{0}\left(\left[\alpha_{\min }, \alpha_{\max }\right]\right)$-hard, then

$$
\left\{x \in \mathbb{R}^{d} \mid \lim _{r \searrow 0} \frac{\log \mu(B(x, r))}{\log r} \in A\right\} \quad \text { is } \quad \Sigma_{\gamma}^{0} \text {-hard } \quad \text { for } 3 \geq \gamma .
$$

This result can be improved for $\gamma=3$. In particular, our first result shows that the multifractal decomposition set $\Delta_{\mu}(A)=\left\{x \in \mathbb{R}^{d} \mid \lim _{r \searrow 0} \frac{\log \mu(B(x, r))}{\log r} \in A\right\}$ of a self-similar measure is always $\Pi_{3}^{0}$-hard for all subsets $A$ of $\left[\alpha_{\min }, \alpha_{\max }\right]$

Theorem 3.2. Let $K$ and $\mu$ be the self-similar sets and self-similar measure in (3.1) and (3.2), respectively, and assume that the $S S C$ is satisfied. Write $s=\operatorname{dim}_{H}(K)$ and assume that $\left(p_{1}, \ldots, p_{N}\right) \neq$ $\left(r_{1}^{s}, \ldots, r_{N}^{s}\right)$. If $A \subseteq\left[\alpha_{\min }, \alpha_{\max }\right]$, then the set

$$
\left\{x \in \mathbb{R}^{d} \mid \lim _{r \searrow 0} \frac{\log \mu(B(x, r))}{\log r} \in A\right\}
$$

is $\Pi_{3}^{0}$-hard.

The proof of Theorem 3.2 is presented in Section 7. The proof is based on establishing the following reduction, namely, if we write $C=\left\{\nu_{1} \nu_{2} \ldots \in \mathbb{N}^{\mathbb{N}} \mid \nu_{n} \rightarrow \infty\right\}$, then

$$
\left(\mathbb{N}^{\mathbb{N}} ; C\right) \underset{\mathrm{w}}{\leq}\left(\mathbb{R}^{d} ; \Delta_{\mu}(A)\right) .
$$

Since $C$ is $\Pi_{3}^{0}$-complete (see, for example, [Ke, Exercise 23.2]), the statement in Theorem 3.2 follows immediately from (3.5).

Next, we discuss completeness of $\Delta_{\mu}(A)=\left\{x \in \mathbb{R}^{d} \mid \lim _{r \searrow 0} \frac{\log \mu(B(x, r))}{\log r} \in A\right\}$ for the self-similar measure $\mu$ in (3.2). As above, it follows from Lemma 3.1 that we can apply Theorem 2.3 to the self-similar measure $\mu$. This leads to to following result. 
Theorem 3.3. Let $K$ and $\mu$ be the self-similar sets and self-similar measure in (3.1) and (3.2), respectively, and assume that the SSC is satisfied. Let $\gamma$ be an ordinate with $\gamma<\omega_{1}$.

(1) If $A \subseteq\left[\alpha_{\min }, \alpha_{\max }\right]$ is $\Pi_{\gamma}^{0}\left(\left[\alpha_{\min }, \alpha_{\max }\right]\right)$-complete, then

$$
\left\{x \in \mathbb{R}^{d} \mid \lim _{r \searrow 0} \frac{\log \mu(B(x, r))}{\log r} \in A\right\} \text { is } \begin{cases}\Pi_{\gamma+1}^{0} \text {-complete } & \text { for } 3 \leq \gamma<\omega \\ \Pi_{\gamma}^{0} \text {-complete } & \text { for } \omega \leq \gamma .\end{cases}
$$

(2) If $A \subseteq\left[\alpha_{\min }, \alpha_{\max }\right]$ is $\Sigma_{\gamma}^{0}\left(\left[\alpha_{\min }, \alpha_{\max }\right]\right)$-complete, then

$$
\left\{x \in \mathbb{R}^{d} \mid \lim _{r \searrow 0} \frac{\log \mu(B(x, r))}{\log r} \in A\right\} \text { is } \begin{cases}\Sigma_{\gamma+1}^{0} \text {-complete } & \text { for } 3 \leq \gamma<\omega \\ \Sigma_{\gamma}^{0} \text {-complete } & \text { for } \omega \leq \gamma\end{cases}
$$

Proof.

This statement follows immediately from Lemma 3.1 and Theorem 2.3.

For particular choices of the set $A$, namely, for $A \in \Pi_{2}^{0}$, the result in Theorem 3.3 can be improved. In particular, for all $A \in \Pi_{2}^{0}$, (regardless of whether or not $A$ is $\Pi_{2}^{0}$-complete), the set $\Delta_{\mu}(A)=\{x \in$ $\left.\mathbb{R}^{d} \mid \lim _{r \searrow 0} \frac{\log \mu(B(x, r))}{\log r} \in A\right\}$ is always $\Pi_{3}^{0}$-complete; this is the statement of Theorem 3.4 below.

Theorem 3.4. Let $K$ and $\mu$ be the self-similar sets and self-similar measure in (3.1) and (3.2), respectively, and assume that the $S S C$ is satisfied. Write $s=\operatorname{dim}_{H}(K)$ and assume that $\left(p_{1}, \ldots, p_{N}\right) \neq$ $\left(r_{1}^{s}, \ldots, r_{N}^{s}\right)$. If $A \subseteq\left[\alpha_{\min }, \alpha_{\max }\right]$ is $\Pi_{2}^{0}$, then the set

$$
\left\{x \in \mathbb{R}^{d} \mid \lim _{r \searrow 0} \frac{\log \mu(B(x, r))}{\log r} \in A\right\}
$$

is $\Pi_{3}^{0}$-complete.

Proof.

Recall that we write $\Delta_{\mu}(A)=\left\{x \in \mathbb{R}^{d} \mid \lim _{r} \backslash 0 \frac{\log \mu(B(x, r))}{\log r} \in A\right\}$. We must now prove that $\Delta_{\mu}(A)$ is $\Pi_{3}^{0}$-hard and that $\Delta_{\mu}(A) \in \Pi_{3}^{0}$. We first note that it follows from Theorem 3.2 that $\Delta_{\mu}(A)$ is $\Pi_{3}^{0}$-hard. Also, since $A \in \Pi_{2}^{0}$, we conclude from Theorem 2.1 that $\Delta_{\mu}(A) \in \Pi_{2+1}^{0}=\Pi_{3}^{0}$.

The following special case of Theorem 3.4, providing information about the classical multifractal decomposition sets, namely, $\Delta_{\mu}(\alpha)=\left\{x \in K \mid \lim _{r \searrow 0} \frac{\log \mu(B(x, r))}{\log r}=\alpha\right\}$ for $\alpha \in \mathbb{R}$, seems worthwhile presenting separately.

Corollary 3.5. Let $K$ and $\mu$ be the self-similar sets and self-similar measure in (3.1) and (3.2), respectively, and assume that the $S S C$ is satisfied. Write $s=\operatorname{dim}_{\mathrm{H}}(K)$ and assume that $\left(p_{1}, \ldots, p_{N}\right) \neq$ $\left(r_{1}^{s}, \ldots, r_{N}^{s}\right)$. If $\alpha \in\left[\alpha_{\min }, \alpha_{\max }\right]$, then the set

$$
\left\{x \in \mathbb{R}^{d} \mid \lim _{r \searrow 0} \frac{\log \mu(B(x, r))}{\log r}=\alpha\right\}
$$

is $\Pi_{3}^{0}$-complete.

Proof.

This follows from Theorem 3.4 since $\{\alpha\} \in \Pi_{2}^{0}$. 
3.3. Divergence points of self-similar measures. Of course, for a Borel measure $\lambda$ on $\mathbb{R}^{d}$, the the limit

$$
\lim _{r \searrow 0} \frac{\log \lambda(B(x, r))}{\log r}
$$

may not exist at all points $x$. Points $x$ for which this limit does not exist are called divergence points, i.e. the set $D_{\lambda}$ of divergence points of the measure $\lambda$ is defined by

$$
D_{\lambda}=\left\{x \in \mathbb{R}^{d} \mid \frac{\log \lambda(B(x, r))}{\log r} \text { diverges as } r \searrow 0\right\} .
$$

The set of divergence points have until very recently been considered of little interest in dynamical systems and geometric measure theory. Indeed, according to folklore, these sets carried no essential information about the underlying structure. However, recently Barriare \& Schmeling [BaSc] have shown that the set of divergence points of self-similar measures typically is highly "visible" or "observable", namely, it has full Hausdorff dimension. More precisely, Barriare \& Schmeling [BaSs] proved the following result. Let $K$ and $\mu$ be the self-similar sets and self-similar measure in (3.1) and (3.2), respectively, and assume that the SSC is satisfied. Write $s=\operatorname{dim}_{\mathrm{H}}(K)$. If $\left(p_{1}, \ldots, p_{N}\right) \neq\left(r_{1}^{s}, \ldots, r_{N}^{s}\right)$, then

$$
\operatorname{dim}_{H}\left\{x \in \mathbb{R}^{d} \mid \frac{\log \mu(B(x, r))}{\log r} \text { diverges as } r \searrow 0\right\}=\operatorname{dim}_{H} K .
$$

Sets of divergence points have been investigated further in, for example, [FaFe,OlWi] showing that the set of divergence points has a surprising rich and intricate fractal structure.

We will now analyse the set-theoretical complexity of the set of divergence points of a self-similar measure $\mu$. We first note that is easy to give an "upper bound" for the position of $D_{\mu}$ in the Borel Hierarchy. Namely, if $\mu$ is the self-similar measure in (3.2) and we assume that the SSC is satisfied, then we have

$$
\left\{\begin{array}{l|l}
x \in \mathbb{R}^{d} & \frac{\log \mu(B(x, r))}{\log r} \text { diverges as } r \searrow 0
\end{array}\right\} \in \Sigma_{3}^{0} ;
$$

indeed, it follows from Lemma 4.3 that if $\lambda$ is aspherical then $D_{\lambda} \in \Sigma_{3}^{0}$, and Lemma 3.1 shows that any self-similar measure satisfying the SSC is aspherical. However, this result can be improved substantially; not only does the set of divergence points of a self-similar measures belong to $\Sigma_{3}^{0}$, it is, in fact, $\Sigma_{3}^{0}-$ complete; this is the contents of the next theorem.

Theorem 3.6. Let $K$ and $\mu$ be the self-similar sets and self-similar measure in (3.1) and (3.2), respectively, and assume that the SSC is satisfied. Write $s=\operatorname{dim}_{\mathrm{H}}(K)$ and assume that $\left(p_{1}, \ldots, p_{N}\right) \neq$ $\left(r_{1}^{s}, \ldots, r_{N}^{s}\right)$. Then the set

$$
\left\{x \in \mathbb{R}^{d} \mid \frac{\log \mu(B(x, r))}{\log r} \text { diverges as } r \searrow 0\right\}
$$

is $\Sigma_{3}^{0}$-complete.

The proof of Theorem 3.6 is given in Section 7. The proof is based on establishing the following reduction, namely, if $C$ denotes the set in (3.5), then

$$
\left(\mathbb{N}^{\mathbb{N}} ; \mathbb{N}^{\mathbb{N}} \backslash C\right) \underset{\mathrm{w}}{\leq}\left(\mathbb{R}^{d} ; D_{\mu}\right)
$$

Since $C$ is $\Pi_{3}^{0}$-complete (see, for example, [Ke, Exercise 23.2]), the statement in Theorem 3.2 follows immediately from (3.6).

Theorem 3.6 may be viewed as yet another manifestation of the fact that the set of divergence points of self-similar measures typically is highly "visible" or "observable". 


\section{Proofs of Theorems 2.1-2.3: Auxiliary Results.}

The purpose of this section is to prove three auxiliary results for aspherical measures; recall that the definition of an aspherical measure is given in Section 2.1.

Lemma 4.1. Let $\lambda$ be a finite Borel measure on $\mathbb{R}^{d}$ and assume that $\lambda$ is aspherical. Then the map defined by

$$
x \rightarrow \lambda(B(x, r))
$$

is continuous for all $r>0$.

Proof.

Recall that for $x \in \mathbb{R}^{d}$ and $r>0$, we write $S(x, r)=\left\{y \in \mathbb{R}^{d}|| x-y \mid=r\right\}$, and note that $\lambda(S(x, r))=0$ for all $x \in \mathbb{R}^{d}$ and all $r>0$ since $\lambda$ is aspherical. Now fix $r>0$. Let $x \in \mathbb{R}^{d}$ and write $E(\delta)=$ $B(x, r+\delta) \backslash B(x, r-\delta)$ for $\delta>0$. Since $E\left(\delta_{1}\right) \subseteq E\left(\delta_{2}\right)$ for $\delta_{1} \leq \delta_{2}$ and $\cap_{\delta>0} E(\delta)=S(x, r)$, we conclude that $\lambda(E(\delta)) \rightarrow \lambda(S(x, r))=0$ as $\delta \searrow 0$. Hence, for $y \in \mathbb{R}^{d}$, we have $|\lambda(B(x, r))-\lambda(B(y, r))| \leq$ $\lambda(B(x, r+|x-y|) \backslash B(x, r-|x-y|))=\lambda(E(|x-y|)) \rightarrow 0$ as $y \rightarrow x$.

Lemma 4.2. Let $\lambda$ be a finite Borel measure on $\mathbb{R}^{d}$.

(1) We have

$$
\left\{x \in \mathbb{R}^{d} \mid \lim _{r \searrow 0} \frac{\log \mu(B(x, r))}{\log r}=\alpha\right\}=\left\{x \in \mathbb{R}^{d} \mid \lim _{n} \frac{\log \mu\left(B\left(x, 2^{-n}\right)\right)}{\log 2^{-n}}=\alpha\right\}
$$

for all $\alpha \in \mathbb{R}$.

(2) If, in addition, $\lambda$ is aspherical, then we have

$$
\left\{x \in \mathbb{R}^{d} \mid \lim _{r \searrow 0} \frac{\log \mu(B(x, r))}{\log r}=\alpha\right\} \in \Pi_{3}^{0}
$$

for all $\alpha \in \mathbb{R}$.

Proof.

(1) Fix $\alpha \in \mathbb{R}$ and let $x \in \mathbb{R}^{d}$. For $r>0$ and $n \in \mathbb{N}$ with $2^{-(n+1)} \leq r \leq 2^{-n}$, it is not difficult to see that $\frac{n}{n+1} \frac{\log \mu\left(B\left(x, 2^{-n}\right)\right)}{\log 2^{-n}} \leq \frac{\log \mu(B(x, r))}{\log r} \leq \frac{n+1}{n} \frac{\log \mu\left(B\left(x, 2^{-(n+1)}\right)\right)}{\log 2^{-(n+1)}}$, whence

$$
\left\{x \in \mathbb{R}^{d} \mid \lim _{r \searrow 0} \frac{\log \mu(B(x, r))}{\log r}=\alpha\right\}=\left\{x \in \mathbb{R}^{d} \mid \lim _{n} \frac{\log \mu\left(B\left(x, 2^{-n}\right)\right)}{\log 2^{-n}}=\alpha\right\} .
$$

(2) Note that it follows from Part (1) that

$$
\begin{aligned}
\left\{x \in \mathbb{R}^{d} \mid \lim _{r \searrow 0} \frac{\log \mu(B(x, r))}{\log r}\right\} & =\left\{x \in \mathbb{R}^{d} \mid \lim _{n} \frac{\log \mu\left(B\left(x, 2^{-n}\right)\right)}{\log 2^{-n}}=\alpha\right\} \\
& =\bigcap_{k \in \mathbb{N}} \bigcup_{N \in \mathbb{N}} \bigcap_{\substack{n \in \mathbb{N} \\
n \geq N}}\left\{x \in \mathbb{R}^{d}|| \frac{\log \mu\left(B\left(x, 2^{-n}\right)\right)}{\log 2^{-n}}-\alpha \mid \leq \frac{1}{k}\right\} .
\end{aligned}
$$

Since Lemma 4.1 implies that the set $\left\{x \in \mathbb{R}^{d}|| \frac{\log \mu\left(B\left(x, r_{n}\right)\right)}{\log r_{n}}-\alpha \mid \leq \frac{1}{k}\right\}$ is closed for all $n, k \in \mathbb{N}$, the desired conclusion follows from (4.1).

Lemma 4.3. Let $\lambda$ be a finite Borel measure on $\mathbb{R}^{d}$ and assume that $\lambda$ is aspherical. Then

$$
\left\{\begin{array}{l|l}
x \in \mathbb{R}^{d} & \frac{\log \lambda(B(x, r))}{\log r} \text { diverges as } r \searrow 0
\end{array}\right\} \in \Sigma_{3}^{0} .
$$


Proof.

Let $x \in \mathbb{R}^{d}$. We first note that it follows from Lemma 4.2 that

$$
\left\{x \in \mathbb{R}^{d} \mid \frac{\log \mu(B(x, r))}{\log r} \text { diverges as } r \searrow 0\right\}=\left\{x \in \mathbb{R}^{d} \mid \frac{\log \mu\left(B\left(x, 2^{-n}\right)\right)}{\log 2^{-n}} \text { diverges as } n \rightarrow \infty\right\},
$$

whence

$$
\begin{aligned}
\left\{x \in \mathbb{R}^{d}\right. & \left.\mid \frac{\log \mu(B(x, r))}{\log r} \text { diverges as } r \searrow 0\right\} \\
& =\left\{x \in \mathbb{R}^{d} \mid\left(\frac{\log \mu\left(B\left(x, 2^{-n}\right)\right)}{\log 2^{-n}}\right)_{n} \text { is not a Cauchy sequence }\right\} \\
& =\bigcup_{k \in \mathbb{N}} \bigcap_{N \in \mathbb{N}} \bigcup_{\substack{n, m \in \mathbb{N} \\
n, m \geq N}}\left\{x \in \mathbb{R}^{d}|| \frac{\log \mu\left(B\left(x, 2^{-n}\right)\right)}{\log 2^{-n}}-\frac{\log \mu\left(B\left(x, 2^{-m}\right)\right)}{\log 2^{-m}} \mid>\frac{1}{k}\right\} .
\end{aligned}
$$

Since it follows from Lemma 4.1 that the set $\left\{x \in \mathbb{R}^{d}|| \frac{\log \mu\left(B\left(x, 2^{-n}\right)\right)}{\log 2^{-n}}-\frac{\log \mu\left(B\left(x, 2^{-m}\right)\right)}{\log 2^{-m}} \mid>\frac{1}{k}\right\}$ is open for all $n, m, k \in \mathbb{N}$, the desired conclusion follows from (4.2).

\section{Proof of Theorem 2.1.}

The purpose of this section is to prove Theorem 2.1. Recall, that for a Borel measure $\lambda$ on $\mathbb{R}^{d}$ and $A \subseteq \mathbb{R}$, we write

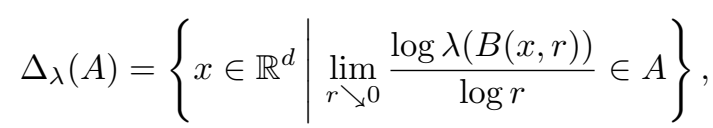

and use the following simplified notation, namely, $\Delta_{\lambda}(\alpha)=\Delta_{\lambda}(\{\alpha\})$ if $A=\{\alpha\}$ is a singleton with $\alpha \in \mathbb{R}$. Finally, recall that we write

$$
D_{\lambda}=\left\{x \in \mathbb{R}^{d} \mid \frac{\log \lambda(B(x, r))}{\log r} \text { diverges as } r \searrow 0\right\} .
$$

We now turn towards the proof of Theorem 2.1.

Proposition 5.1. Let $\lambda$ be a finite Borel measure on $\mathbb{R}^{d}$ and assume that $\lambda$ is aspherical. Let $\gamma$ be an ordinal with $3 \leq \gamma<\omega_{1}$.

(1) If $A \in \Pi_{\kappa}^{0}$ for some $\kappa<\gamma$, then there is a set $L \in \Pi_{\gamma}^{0}$ such that $\Delta_{\lambda}(A)=\left(\mathbb{R}^{d} \backslash D_{\lambda}\right) \cap L$.

(2) If $A \in \Sigma_{\kappa}^{0}$ for some $\kappa<\gamma$, then there is a set $M \in \Sigma_{\gamma}^{0}$ such that $\Delta_{\lambda}(A)=\left(\mathbb{R}^{d} \backslash D_{\lambda}\right) \cap M$.

Proof.

For an ordinal $\gamma$ with $3 \leq \gamma<\omega_{1}$, let $P(\gamma)$ denote the statement:

(1) If $A \in \Pi_{\kappa}^{0}$ for some $\kappa<\gamma$, then there is a set $L \in \Pi_{\gamma}^{0}$ such that $\Delta_{\lambda}(A)=\left(\mathbb{R}^{d} \backslash D_{\lambda}\right) \cap L$,

and

(2) If $A \in \Sigma_{\kappa}^{0}$ for some $\kappa<\gamma$, then there is a set $M \in \Sigma_{\gamma}^{0}$ such that $\Delta_{\lambda}(A)=\left(\mathbb{R}^{d} \backslash D_{\lambda}\right) \cap M$.

We will now prove that $P(\gamma)$ is true for all ordinals $\gamma$ with $3 \leq \gamma<\omega_{1}$ by transfinite induction.

The start of the induction.

We now prove that statements (1) and (2) in $P(\gamma)$ are true for $\gamma=3$. 
Proof of statement (1) in $P(3)$. Let $A \in \Pi_{\kappa}^{0}$ for $\kappa<3$. In particular, we conclude that $A \in \Pi_{\kappa}^{0} \subseteq \Pi_{2}^{0}$ and we can therefore find a sequence $\left(G_{i}\right)_{i}$ of open sets such that $A=\cap_{i} G_{i}$. Now put

$$
L=\bigcap_{i \in \mathbb{N}} \bigcup_{\substack{q, r \in \mathbb{Q} \\ r>0}} \bigcup_{\substack{N \in \mathbb{N} \\ B(q, r) \subseteq G_{i}}} \bigcap_{\substack{n \in \mathbb{N} \\ n \geq N}}\left\{x \in \mathbb{R}^{d} \mid \frac{\log \lambda\left(B\left(x, 2^{-n}\right)\right)}{\log 2^{-n}} \in \overline{B(q, r)}\right\} .
$$

It follows from Lemma 4.1 that the set $\left\{x \in \mathbb{R}^{d} \mid \frac{\log \lambda\left(B\left(x, 2^{-n}\right)\right)}{\log 2^{-n}} \in \overline{B(q, r)}\right\}$ is closed for all $q, r \in \mathbb{Q}$ and all $n \in \mathbb{N}$, and we therefore conclude that $L \in \Pi_{3}^{0}$. Also, it is not difficult to see that

$$
\Delta_{\lambda}(A)=\left(\mathbb{R}^{d} \backslash D_{\lambda}\right) \cap L .
$$

This completes the proof of statement (1) in $P(3)$.

Proof of statement (2) in $P(3)$. Let $A \in \Sigma_{\kappa}^{0}$ for $\kappa<3$. In particular, we deduce that $\mathbb{R} \backslash A \in \Pi_{\kappa}^{0}$, and since $\kappa<3$, it therefore follows from statement (1) in $P(3)$ (recall that statement (1) in $P(3)$ was proven above), that there is a set $L \in \Pi_{\gamma}^{0}$ such that

$$
\Delta_{\lambda}(\mathbb{R} \backslash A)=\left(\mathbb{R}^{d} \backslash D_{\lambda}\right) \cap L .
$$

Since $L \in \Pi_{\gamma}^{0}$, it follows that $M=\mathbb{R} \backslash L \in \Sigma_{\gamma}^{0}$, and (5.1) implies that

$$
\begin{aligned}
\Delta_{\lambda}(A) & =\left(\mathbb{R}^{d} \backslash D_{\lambda}\right) \backslash \Delta_{\lambda}(\mathbb{R} \backslash A) \\
& =\left(\mathbb{R}^{d} \backslash D_{\lambda}\right) \cap\left(\mathbb{R}^{d} \backslash L\right) \\
& =\left(\mathbb{R}^{d} \backslash D_{\lambda}\right) \cap M .
\end{aligned}
$$

This completes the proof of statement (2) in $P(3)$.

The inductive step.

Let $\gamma>3$ and assume that statements (1) and $(2)$ in $P(\zeta)$ are true for all $\zeta<\gamma$. We will now prove that statements (1) and (2) in $P(\gamma)$ are true.

Proof of statement (1) in $P(\gamma)$. Let $A \in \Pi_{\kappa}^{0}$ for $\kappa<\gamma$. We must now show that there is a set $L \in \Pi_{\gamma}^{0}$ such that $\Delta_{\lambda}(A)=\left(\mathbb{R}^{d} \backslash D_{\lambda}\right) \cap L$. Since $A \in \Pi_{\kappa}^{0}$, we can find a sequence $\left(\kappa_{n}\right)_{n}$ of ordinals with $\kappa_{n}<\kappa$ and a sequence $\left(A_{n}\right)_{n}$ of sets with $A_{n} \in \Sigma_{\kappa_{n}}^{0}$ such that $A=\cap_{n} A_{n}$. Since $\kappa<\gamma$, we conclude that statement (2) in $P(\kappa)$ is true, and because $\kappa_{n}<\kappa$ and $A_{n} \in \Sigma_{\kappa_{n}}^{0}$, it therefore follows from statement (2) in $P(\kappa)$ that there is a set $M_{n} \in \Sigma_{\kappa}^{0}$ such that

$$
\Delta_{\lambda}\left(A_{n}\right)=\left(\mathbb{R}^{d} \backslash D_{\lambda}\right) \cap M_{n} .
$$

Now put $L=\cap_{n} M_{n}$. Since $M_{n} \in \Sigma_{\kappa}^{0}$ and $\kappa<\gamma$, we see that $L=\cap_{n} M_{n} \in \Pi_{\gamma}^{0}$. Also

$$
\begin{aligned}
\Delta_{\lambda}(A) & =\Delta_{\lambda}\left(\cap_{n} A_{n}\right) \\
& =\bigcap_{n} \Delta_{\lambda}\left(A_{n}\right) \\
& =\left(\mathbb{R}^{d} \backslash D_{\lambda}\right) \cap \bigcap_{n} M_{n} \\
& =\left(\mathbb{R}^{d} \backslash D_{\lambda}\right) \cap L .
\end{aligned}
$$

This completes the proof of statement (1) in $P(\gamma)$.

Proof of statement (2) in $P(\gamma)$. Let $A \in \Sigma_{\kappa}^{0}$ for $\kappa<\gamma$. We must now show that there is a set $M \in \Sigma_{\gamma}^{0}$ such that $\Delta_{\lambda}(A)=\left(\mathbb{R}^{d} \backslash D_{\lambda}\right) \cap M$. Since $A \in \Sigma_{\kappa}^{0}$, we can find a sequence $\left(\kappa_{n}\right)_{n}$ of ordinals with $\kappa_{n}<\kappa$ and a sequence $\left(A_{n}\right)_{n}$ of sets with $A_{n} \in \Pi_{\kappa_{n}}^{0}$ such that $A=\cup_{n} A_{n}$. Since $\kappa<\gamma$, we conclude that statement (1) in $P(\kappa)$ is true, and because $\kappa_{n}<\kappa$ and $A_{n} \in \Pi_{\kappa_{n}}^{0}$, it therefore follows from statement (2) in $P(\kappa)$ that there is a set $L_{n} \in \Sigma_{\kappa}^{0}$ such that

$$
\Delta_{\lambda}\left(A_{n}\right)=\left(\mathbb{R}^{d} \backslash D_{\lambda}\right) \cap L_{n} .
$$


Now put $M=\cup_{n} L_{n}$. Since $L_{n} \in \Pi_{\kappa}^{0}$ and $\kappa<\gamma$, we see that $M=\cup_{n} L_{n} \in \Sigma_{\gamma}^{0}$. Also

$$
\begin{aligned}
\Delta_{\lambda}(A) & =\Delta_{\lambda}\left(\cup_{n} A_{n}\right) \\
& =\bigcup_{n} \Delta_{\lambda}\left(A_{n}\right) \\
& =\left(\mathbb{R}^{d} \backslash D_{\lambda}\right) \cap \bigcup_{n} L_{n} \\
& =\left(\mathbb{R}^{d} \backslash D_{\lambda}\right) \cap M .
\end{aligned}
$$

This completes the proof of statement $(2)$ in $P(\gamma)$.

Proposition 5.2. Let $\lambda$ be a finite Borel measure on $\mathbb{R}^{d}$ and assume that $\lambda$ is aspherical. Let $\gamma$ be an ordinal with $3 \leq \gamma<\omega_{1}$.

(1) Assume $\gamma \geq 3$. If $A \in \Pi_{\kappa}^{0}$ for some $\kappa<\gamma$, then $\Delta_{\lambda}(A) \in \Pi_{\gamma}^{0}$.

(2) Assume $\gamma \geq 4$. If $A \in \Sigma_{\kappa}^{0}$ for some $\kappa<\gamma$, then $\Delta_{\lambda}(A) \in \Sigma_{\gamma}^{0}$.

Proof.

(1) It follows from Proposition 5.1 that there is a set $L \in \Pi_{\gamma}^{0}$ such that $\Delta_{\lambda}(A)=\left(\mathbb{R}^{d} \backslash D_{\lambda}\right) \cap L$. Since $D_{\lambda} \in \Sigma_{3}^{0}$ (by Lemma 4.3) and $\gamma \geq 3$, we conclude that $\mathbb{R}^{d} \backslash D_{\lambda} \in \Pi_{3}^{0} \subseteq \Pi_{\gamma}^{0}$. It follows from this that $\Delta_{\lambda}(A)=\left(\mathbb{R}^{d} \backslash D_{\lambda}\right) \cap L \in \Pi_{\gamma}^{0}$.

(2) It follows from Proposition 5.1 that there is a set $M \in \Sigma_{\gamma}^{0}$ such that $\Delta_{\lambda}(A)=\left(\mathbb{R}^{d} \backslash D_{\lambda}\right) \cap M$. Since $D_{\lambda} \in \Sigma_{3}^{0}$ (by Lemma 4.1) and $\gamma \geq 4$, we conclude that $\mathbb{R}^{d} \backslash D_{\lambda} \in \Pi_{3}^{0} \subseteq \Sigma_{\gamma}^{0}$. It follows from this that $\Delta_{\lambda}(A)=\left(\mathbb{R}^{d} \backslash D_{\lambda}\right) \cap L \in \Sigma_{\gamma}^{0}$.

Proposition 5.3. Let $\lambda$ be a finite Borel measure on $\mathbb{R}^{d}$ and assume that $\lambda$ is aspherical. Let $\gamma$ be an ordinal with $\omega \leq \gamma<\omega_{1}$.

(1) If $A \in \Pi_{\gamma}$, then $\Delta_{\lambda}(A) \in \Pi_{\gamma}^{0}$.

(2) If $A \in \Sigma_{\gamma}$, then $\Delta_{\lambda}(A) \in \Sigma_{\gamma}^{0}$.

Proof.

For an ordinal $\gamma$ with $\omega \leq \gamma<\omega_{1}$, let $P(\gamma)$ denote the statement:

(1) If $A \in \Pi_{\gamma}$, then $\Delta_{\lambda}(A) \in \Pi_{\gamma}^{0}$, and

(2) If $A \in \Sigma_{\gamma}$, then $\Delta_{\lambda}(A) \in \Sigma_{\gamma}^{0}$.

We will now prove that $P(\gamma)$ is true for all ordinals $\gamma$ with $\omega \leq \gamma<\omega_{1}$ by transfinite induction.

The start of the induction.

We will now prove that statements (1) and $(2)$ in $P(\gamma)$ are true for $\gamma=\omega$.

Proof of statement (1) in $P(\omega)$. Let $A \in \Pi_{\omega}^{0}$. We must now show that $\Delta_{\lambda}(A) \in \Pi_{\omega}^{0}$. Since $A \in \Pi_{\omega}^{0}$, we can find a sequence $\left(\kappa_{n}\right)_{n}$ of ordinals with $\kappa_{n}<\omega$ and a sequence $\left(A_{n}\right)_{n}$ of sets with $A_{n} \in \Sigma_{\kappa_{n}}^{0}$ such that $A=\cap_{n} A_{n}$. Also, since $A_{n} \in \Sigma_{\kappa_{n}}^{0}$ and $\kappa_{n}<\left(\kappa_{n} \vee 4\right)+1$ with $\left(\kappa_{n} \vee 4\right)+1 \geq 4$, it now follows from Proposition 5.2 that $\Delta_{\lambda}\left(A_{n}\right) \in \Sigma_{\left(\kappa_{n} \vee 4\right)+1}^{0}$. Finally, as $\left(\kappa_{n} \vee 4\right)+1<\omega$ (because $\left.\kappa_{n}<\omega\right)$, this shows that $\cap_{n} \Delta_{\lambda}\left(A_{n}\right) \in \Pi_{\omega}^{0}$, and so $\Delta_{\lambda}(A)=\Delta_{\lambda}\left(\cap_{n} A_{n}\right)=\cap_{n} \Delta_{\lambda}\left(A_{n}\right) \in \Pi_{\omega}^{0}$. This completes the proof of statement $(1)$ in $P(\omega)$.

Proof of statement (2) in $P(\omega)$. Let $A \in \Sigma_{\omega}^{0}$. We must now show that $\Delta_{\lambda}(A) \in \Sigma_{\omega}^{0}$. Since $A \in \Sigma_{\omega}^{0}$, we can find a sequence $\left(\kappa_{n}\right)_{n}$ of ordinals with $\kappa_{n}<\omega$ and a sequence $\left(A_{n}\right)_{n}$ of sets with $A_{n} \in \Pi_{\kappa_{n}}^{0}$ such that $A=\cup_{n} A_{n}$. Also, since $A_{n} \in \Pi_{\kappa_{n}}^{0}$ and $\kappa_{n}<\left(\kappa_{n} \vee 3\right)+1$ with $\left(\kappa_{n} \vee 3\right)+1 \geq 3$, it now follows from Proposition 5.2 that $\Delta_{\lambda}\left(A_{n}\right) \in \Pi_{\left(\kappa_{n} \vee 3\right)+1}^{0}$. Finally, as $\left(\kappa_{n} \vee 3\right)+1<\omega$ (because $\left.\kappa_{n}<\omega\right)$, this shows that $\cap_{n} \Delta_{\lambda}\left(A_{n}\right) \in \Pi_{\omega}^{0}$, and so $\Delta_{\lambda}(A)=\Delta_{\lambda}\left(\cup_{n} A_{n}\right)=\cup_{n} \Delta_{\lambda}\left(A_{n}\right) \in \Sigma_{\omega}^{0}$. This completes the proof of statement (2) in $P(\omega)$. 
The inductive step.

Let $\gamma>\omega$ and assume that statements (1) and (2) in $P(\zeta)$ are true for all $\omega \leq \zeta<\gamma$. We will now prove that statements (1) and (2) in $P(\gamma)$ are true.

Proof of statement (1) in $P(\gamma)$. Let $A \in \Pi_{\gamma}^{0}$. We must now show that $\Delta_{\lambda}(A) \in \Pi_{\gamma}^{0}$. Since $A \in \Pi_{\gamma}^{0}$, we can find a sequence $\left(\kappa_{n}\right)_{n}$ of ordinals with $\kappa_{n}<\gamma$ and a sequence $\left(A_{n}\right)_{n}$ of sets with $A_{n} \in \Sigma_{\kappa_{n}}^{0}$ such that $A=\cap_{n} A_{n}$. Also, since $\omega \leq\left(\kappa_{n} \vee \omega\right)<\gamma$, we conclude that statement (2) in $P\left(\kappa_{n} \vee \omega\right)$ is true, and because $A_{n} \in \Sigma_{\kappa_{n}}^{0} \subseteq \Sigma_{\kappa_{n} \vee \omega}^{0}$, it therefore follows from statement (2) in $P\left(\kappa_{n} \vee \omega\right)$ that $\Delta_{\lambda}\left(A_{n}\right) \in \Sigma_{\kappa_{n} \vee \omega}^{0}$. Finally, as $\left(\kappa_{n} \vee \omega\right)<\gamma$ (because $\kappa_{n}<\gamma$ ), this shows that $\cap_{n} \Delta_{\lambda}\left(A_{n}\right) \in \Pi_{\gamma}^{0}$, and so

$$
\begin{aligned}
\Delta_{\lambda}(A) & =\Delta_{\lambda}\left(\cap_{n} A_{n}\right) \\
& =\bigcap_{n} \Delta_{\lambda}\left(A_{n}\right) \\
& \in \Pi_{\omega}^{0} .
\end{aligned}
$$

This completes the proof of statement (1) in $P(\gamma)$.

Proof of statement (2) in $P(\gamma)$. Let $A \in \Sigma_{\gamma}^{0}$. We must now show that $\Delta_{\lambda}(A) \in \Sigma_{\gamma}^{0}$. Since $A \in \Sigma_{\gamma}^{0}$, we can find a sequence $\left(\kappa_{n}\right)_{n}$ of ordinals with $\kappa_{n}<\gamma$ and a sequence $\left(A_{n}\right)_{n}$ of sets with $A_{n} \in \Pi_{\kappa_{n}}^{0}$ such that $A=\cup_{n} A_{n}$. Also, since $\omega \leq\left(\kappa_{n} \vee \omega\right)<\gamma$, we conclude that statement (1) in $P\left(\kappa_{n} \vee \omega\right)$ is true, and because $A_{n} \in \Pi_{\kappa_{n}}^{0} \subseteq \Pi_{\kappa_{n} \vee \omega}^{0}$, it therefore follows from statement $(1)$ in $P\left(\kappa_{n} \vee \omega\right)$ that $\Delta_{\lambda}\left(A_{n}\right) \in \Pi_{\kappa_{n} \vee \omega}^{0}$. Finally, as $\left(\kappa_{n} \vee \omega\right)<\gamma$ (because $\kappa_{n}<\gamma$ ), this shows that $\cup_{n} \Delta_{\lambda}\left(A_{n}\right) \in \Sigma_{\gamma}^{0}$, and so

$$
\begin{aligned}
\Delta_{\lambda}(A) & =\Delta_{\lambda}\left(\cup_{n} A_{n}\right) \\
& =\bigcup_{n} \Delta_{\lambda}\left(A_{n}\right) \\
& \in \Sigma_{\omega}^{0} .
\end{aligned}
$$

This completes the proof of statement (2) in $P(\gamma)$.

We can now prove Theorem 2.1.

Proof of Theorem 2.1.

The statement in Theorem 2.1 follows immediately from Proposition 5.2 and Proposition 5.3. 


\section{Proofs of Theorems $2.2-2.3$}

The purpose of this section is to prove Theorem 2.2 and Theorem 2.3. Recall, that for a Borel measure $\lambda$ on $\mathbb{R}^{d}$ and $A \subseteq \mathbb{R}$, we write

$$
\Delta_{\lambda}(A)=\left\{x \in \mathbb{R}^{d} \mid \lim _{r \searrow 0} \frac{\log \lambda(B(x, r))}{\log r} \in A\right\},
$$

and that we use the following simplified notation, namely, $\Delta_{\lambda}(\alpha)=\Delta_{\lambda}(\{\alpha\})$ if $A=\{\alpha\}$ is a singleton with $\alpha \in \mathbb{R}$. Also, recall that we put

$$
I_{\lambda}=\left\{\alpha \in \mathbb{R} \mid \Delta_{\lambda}(\alpha) \neq \varnothing\right\}
$$

6.1. The proof of Theorem 2.2. We first prove Theorem 2.2. Let II denote the set of irrational numbers, and note that it is well-known that $\mathbb{N}^{\mathbb{N}}$ and $[0,1] \cap \mathbb{I}$ are homeomorphic, i.e. there is a homeomorphism $\Phi: \mathbb{N}^{\mathbb{N}} \rightarrow[0,1] \cap \mathbb{I}$ between $\mathbb{N}^{\mathbb{N}}$ and $[0,1] \cap \mathbb{I}$; for example, we may define $\Phi: \mathbb{N}^{\mathbb{N}} \rightarrow \mathbb{R}$ by

$$
\Phi(\boldsymbol{\nu})=\lim _{n} \frac{1}{\nu_{1}+\frac{1}{\nu_{2}+\cdots \frac{1}{\nu_{n-1}+\frac{1}{\nu_{n}}}}}
$$

for $\boldsymbol{\nu}=\nu_{1} \nu_{2} \ldots \in \mathbb{N}^{\mathbb{N}}$. Also, recall that (Wadge) reducibility (denoted by $(X ; A) \leq \underset{\mathrm{w}}{\leq}(Y ; P, Q)$ ) and hardness are defined in Section 1.2.

Proposition 6.1. Let $\lambda$ be a finite Borel measure on $\mathbb{R}^{d}$ and assume that $\lambda$ has a continuous selector and that $\lambda$ is admissible; in particular, there is a subset $U$ of $I_{\lambda}$ such that $I_{\lambda} \backslash U$ is countable and a function $h:[0,1] \cap \mathbb{I} \rightarrow U$ such that $h$ is a homeomorphism between $[0,1] \cap \mathbb{I}$ and $U$. Let $\gamma$ be an ordinal with $\gamma<\omega_{1}$.

(1) If $(h \Phi)^{-1}(A \cap U)$ is $\Pi_{\gamma}^{0}$-hard, then $\Delta_{\lambda}(A)$ is $\Pi_{\gamma}^{0}$-hard for $\gamma \geq 1$.

(2) If $(h \Phi)^{-1}(A \cap U)$ is $\Sigma_{\gamma}^{0}$-hard, then $\Delta_{\lambda}(A)$ is $\Sigma_{\gamma}^{0}$-hard for $\gamma \geq 1$.

Proof.

We first prove the following two claims.

Claim 1. For $N \subseteq \mathbb{N}^{\mathbb{N}}$, we have $\left(\mathbb{N}^{\mathbb{N}} ; N\right) \leq \underset{\mathrm{w}}{ }\left(\mathbb{R}^{d} ; \Delta_{\lambda}(h \Phi(N)), \Delta_{\lambda}\left(h \Phi\left(\mathbb{N}^{\mathbb{N}} \backslash N\right)\right)\right)$.

Proof of Claim 1. We must show that there is a continuous function $f: \mathbb{N}^{\mathbb{N}} \rightarrow \mathbb{R}^{d}$ such that $f(N) \subseteq$ $\Delta_{\lambda}(h \Phi(N))$ and $f\left(\mathbb{N}^{\mathbb{N}} \backslash N\right) \subseteq \Delta_{\lambda}(h \Phi(N))$. Since $\lambda$ has a continuous selector, there is a continuous map $\mathcal{S}: I_{\lambda} \rightarrow \mathbb{R}^{d}$ such that $\mathcal{S}(\alpha) \in \Delta_{\lambda}(\alpha)$ for all $\alpha \in I_{\lambda}$. Now define $f: \mathbb{N}^{\mathbb{N}} \rightarrow \mathbb{R}^{d}$ by $f=\mathcal{S} \circ h \circ \Phi$.

Since $\mathcal{S}, h$ and $\Phi$ are continues, we conclude that $f$ is continuous.

Next, we show that $f(N) \subseteq \Delta_{\lambda}(h \Phi(N))$. Indeed, if $\boldsymbol{\nu} \in N$, then $f(\boldsymbol{\nu})=\mathcal{S}(h(\Phi(\boldsymbol{\nu}))) \in \Delta_{\lambda}(h \Phi(\boldsymbol{\nu})) \subseteq$ $\Delta_{\lambda}(h \Phi(N))$, whence $f(N) \subseteq \Delta_{\lambda}(h \Phi(N))$.

Finally, we show that $f\left(\mathbb{N}^{\mathbb{N}} \backslash N\right) \subseteq \Delta_{\lambda}(h \Phi(N))$. If $\boldsymbol{\nu} \in \mathbb{N}^{\mathbb{N}} \backslash N$, then $f(\boldsymbol{\nu})=\mathcal{S}(h(\Phi(\boldsymbol{\nu}))) \in$ $\Delta_{\lambda}(h \Phi(\boldsymbol{\nu})) \subseteq \Delta_{\lambda}\left(h \Phi\left(\mathbb{N}^{\mathbb{N}} \backslash N\right)\right)$, whence $f\left(\mathbb{N}^{\mathbb{N}} \backslash N\right) \subseteq \Delta_{\lambda}(h \Phi(N))$. This completes the proof of Claim 1 .

Claim 2. For $A \subseteq \mathbb{R}$, we have $\left(\mathbb{N}^{\mathbb{N}} ;(h \Phi)^{-1}(A \cap U)\right) \leq\left(\mathbb{R}^{d} ; \Delta_{\lambda}(A), \Delta_{\lambda}(\mathbb{R} \backslash A)\right)$.

Proof of Claim 2. Applying the statement in Part (1) to the set $N=(h \Phi)^{-1}(A \cap U)$ noticing that in this case $h \Phi(N)=A \cap U$ and $h \Phi\left(\mathbb{N}^{\mathbb{N}} \backslash N\right)=\left(\mathbb{R}^{d} \backslash A\right) \cap U$, we conclude that

$$
\left(\mathbb{N}^{\mathbb{N}} ;(h \Phi)^{-1}(A \cap U)\right) \underset{\mathrm{w}}{\leq}\left(\mathbb{R}^{d} ; \Delta_{\lambda}(A \cap U), \Delta_{\lambda}((\mathbb{R} \backslash A) \cap U)\right) .
$$


However, since $\Delta_{\lambda}(A \cap U) \subseteq \Delta_{\lambda}(A)$ and $\Delta_{\lambda}((\mathbb{R} \backslash A) \cap U) \subseteq \Delta_{\lambda}(\mathbb{R} \backslash A)$ and $\Delta_{\lambda}(A) \cap \Delta_{\lambda}(\mathbb{R} \backslash A)=\varnothing$, it follows from (6.2) that

$$
\left(\mathbb{N}^{\mathbb{N}} ;(h \Phi)^{-1}(A \cap U)\right) \underset{\mathrm{w}}{\leq}\left(\mathbb{R}^{d} ; \Delta_{\lambda}(A), \Delta_{\lambda}(\mathbb{R} \backslash A)\right) .
$$

This completes the proof of Claim 2.

It follows immediately from Claim 2 that if $A \subseteq \mathbb{R}$, then we have

$$
\left(\mathbb{N}^{\mathbb{N}} ;(h \Phi)^{-1}(A \cap U)\right) \underset{\mathrm{w}}{\leq}\left(\mathbb{R}^{d} ; \Delta_{\lambda}(A)\right) .
$$

The statements in the (1) and (2) follow immediately from (6.3).

Proposition 6.2. Let $\lambda$ be a finite Borel measure on $\mathbb{R}^{d}$ and assume that $\lambda$ has a continuous selector and that $\lambda$ is admissible; in particular, there is a subset $U$ of $I_{\lambda}$ such that $I_{\lambda} \backslash U$ is countable and a function $h:[0,1] \cap \mathbb{I} \rightarrow U$ such that $h$ is a homeomorphism between $[0,1] \cap \mathbb{I}$ and $U$. Let $\gamma$ be an ordinal with $\gamma<\omega_{1}$.

(1) If $A \subseteq I_{\lambda}$ and $A$ is $\Pi_{\gamma}^{0}$-hard, then $(h \Phi)^{-1}(A \cap U)$ is $\Pi_{\gamma}^{0}$-hard for $\gamma \geq 3$.

(2) If $A \subseteq I_{\lambda}$ and $A$ is $\Sigma_{\gamma}^{0}$-hard, then $(h \Phi)^{-1}(A \cap U)$ is $\Sigma_{\gamma}^{0}$-hard for $\gamma \geq 3$.

Proof.

For an ordinal $\gamma$ with $\gamma \geq 3$, let $\Gamma$ denote $\Pi_{\gamma}^{0}$ or let $\Gamma$ denote $\Sigma_{\gamma}^{0}$. Let $A$ be a subset of $I_{\lambda}$ and assume that $A$ is $\Gamma(\mathbb{R})$-hard. We now prove the following three claims.

Claim 1. $\mathbb{R} \backslash A \notin \Gamma(\mathbb{R})$.

Proof of Claim 1. This is clear since $A$ is $\Gamma(\mathbb{R})$-hard. This completes the proof of Claim 1.

Claim 2. $\mathbb{R} \backslash(A \cap U) \notin \Gamma(\mathbb{R})$.

Proof of Claim 2. Assume, in order to reach a contradiction, that $\mathbb{R} \backslash(A \cap U) \in \Gamma(\mathbb{R})$. We have

$$
\mathbb{R} \backslash A=(\mathbb{R} \backslash(A \cap U)) \cup(\mathbb{R} \backslash(A \backslash U)),
$$

where, by assumption,

$$
\mathbb{R} \backslash(A \cap U) \in \Gamma(\mathbb{R}) .
$$

Also, since $A \backslash U$ is countable (because $A \backslash U \subseteq I_{\lambda} \backslash U$ and $I_{\lambda} \backslash U$ is countable), we conclude that

$$
\mathbb{R} \backslash(A \backslash U) \in \mathcal{G}_{\delta}(\mathbb{R}) \subseteq \Gamma(\mathbb{R}) .
$$

Combining (6.3), (6.4) and (6.5), we conclude that $\mathbb{R} \backslash A \in \Gamma(\mathbb{R})$ contradicting Claim 1 . This completes the proof of Claim 2.

Claim 3. $U \backslash(A \cap U) \notin \Gamma(U)$.

Proof of Claim 3. For brevity write $M=\mathbb{R} \backslash(A \cap U) \in \Gamma(\mathbb{R})$ and note that $M \cap U=U \backslash(A \cap U)$.

Assume now, in order to reach a contradiction, that $M \cap U=U \backslash(A \cap U) \in \Gamma(U)$. We can therefore find $E \in \Gamma(\mathbb{R})$ such that $M \cap U=E \cap U$, whence

$$
\begin{aligned}
\mathbb{R} \backslash(A \cap U) & =M \\
& =(M \cap U) \cup(M \backslash U) \\
& =(E \cap U) \cup(M \backslash U) \\
& =(E \cap U) \cup(\mathbb{R} \backslash U) \\
& =E \cup(\mathbb{R} \backslash U) .
\end{aligned}
$$

Since $U$ is a homeomorphic image of $[0,1] \cap \mathbb{I}$, we deduce that $U \in \mathcal{G}_{\delta}(\mathbb{R})$, whence $\mathbb{R} \backslash U \in \Gamma(\mathbb{R})$. Also, by assumption, $E \in \Gamma(\mathbb{R})$. Since $\mathbb{R} \backslash U, E \in \Gamma(\mathbb{R})$, we deduce that from (6.6) that $\mathbb{R} \backslash(A \cap U)=$ $E \cup(\mathbb{R} \backslash U) \in \Gamma(\mathbb{R})$ contradicting Claim 2. This completes the proof of Claim 3. 
iu

Using the fact that $h \Phi$ is a homeomorphism between $\mathbb{N}^{\mathbb{N}}$ and $U$, we conclude from Claim 3 that

$$
\mathbb{N}^{\mathbb{N}} \backslash(h \Phi)^{-1}(A \cap U)=(h \Phi)^{-1}(U \backslash(A \cap U)) \notin \Gamma\left(\mathbb{N}^{\mathbb{N}}\right) .
$$

Finally, since $\mathbb{N}^{\mathbb{N}}$ is a zero-dimensional Polish space, it follows from (6.7) and [Ke, Theorem 22.10] that $(h \Phi)^{-1}(A \cap U)$ is $\Gamma\left(\mathbb{N}^{\mathbb{N}}\right)$-hard.

We can now prove Theorem 2.2. For the benefit of the reader we first recall the statement of Theorem 2.2 .

Theorem 2.2. Lower bounds for arbitrary measures: hardness. Let $\lambda$ be a Borel measure on $\mathbb{R}^{d}$ and that $\lambda$ has a continuous selector and that $\lambda$ is admissible. Let $\gamma$ be an ordinal with $\gamma<\omega_{1}$.

(1) If $A \subseteq I_{\lambda}$ is $\Pi_{\gamma}^{0}$-hard, then $\Delta_{\lambda}(A)$ is $\Pi_{\gamma}^{0}$-hard for $\gamma \geq 3$.

(2) If $A \subseteq I_{\lambda}$ is $\Sigma_{\gamma}^{0}$-hard, then $\Delta_{\lambda}(A)$ is $\Sigma_{\gamma}^{0}$-hard for $\gamma \geq 3$.

Proof.

The statement in Theorem 2.2 follows immediately by combining Proposition 6.1 and Proposition 6.2.

6.2. The proof of Theorem 2.3. Next, we turn towards the proof of Theorem 2.3 The proof of Theorem 2.3 relies on the following result by Louveau \& Saint-Raymond [LoSRa].

Theorem 6.3 [LoSRa]. There is a continuous function

$$
\rho:\{0,1\}^{\mathbb{N}} \rightarrow\{0,1\}^{\mathbb{N}},
$$

such that if the subsets $H_{1}, H_{2}, \ldots$ of $\{0,1\}^{\mathbb{N}}$ are defined by

$$
\begin{aligned}
H_{1} & =\{\mathbf{0}\}, \\
H_{n+1} & =\rho^{-1}\left(H_{n}\right) \text { for } n \geq 1,
\end{aligned}
$$

where $\mathbf{0}=000 \ldots$, then $H_{n}$ is $\Pi_{n}^{0}$-complete for all $n$.

Proposition 6.4. Let $\lambda$ be a Borel measure on $\mathbb{R}^{d}$ and assume that $\lambda$ has a continuous selector and that $\lambda$ is admissible. Let $\rho:\{0,1\}^{\mathbb{N}} \rightarrow\{0,1\}^{\mathbb{N}}$ be the map in (6.8). If $H \subseteq\{0,1\}^{\mathbb{N}}$ and $A \subseteq I_{\lambda}$ satisfy

$$
\left(\{0,1\}^{\mathbb{N}} ; H\right) \leq \underset{\mathrm{w}}{\leq}\left(I_{\lambda} ; A\right)
$$

then

$$
\left(\{0,1\}^{\mathbb{N}} ; \rho^{-1}(H)\right) \underset{\mathrm{w}}{\leq}\left(\mathbb{R} ; \Delta_{\lambda}(A), \Delta_{\lambda}(\mathbb{R} \backslash A)\right)
$$

Proof.

We must show that there is a continuous map $f:\{0,1\}^{\mathbb{N}} \rightarrow \mathbb{R}$ such that $f\left(\rho^{-1}(H)\right) \subseteq \Delta_{\lambda}(A)$ and $f\left(\{0,1\}^{\mathbb{N}} \backslash \rho^{-1}(H)\right) \subseteq \Delta_{\lambda}(\mathbb{R} \backslash A)$. By assumption there is a continuous map $\varphi:\{0,1\}^{\mathbb{N}} \rightarrow I_{\lambda}$ such that $\varphi(H) \subseteq A$ and $h \varphi\left(\{0,1\}^{\mathbb{N}} \backslash H\right) \subseteq I_{\lambda} \backslash A$. Also, since $\lambda$ has a continuous selector, there is a continuous map $\mathcal{S}: I_{\lambda} \rightarrow \mathbb{R}^{d}$ such that $\mathcal{S}(\alpha) \in \Delta_{\lambda}(\alpha)$ for all $\alpha \in I_{\lambda}$. Now define $f:\{0,1\}^{\mathbb{N}} \rightarrow \mathbb{R}$ by $f=\mathcal{S} \circ \varphi \circ \rho$.

Since $\mathcal{S}, \varphi$ and $\rho$ are continuous, we conclude that $f$ is continuous.

Next, we show that $f\left(\rho^{-1}(H)\right) \subseteq \Delta_{\lambda}(A)$. Indeed, if $\mathbf{u} \in \rho^{-1}(H)$, then $f(\mathbf{u})=\mathcal{S}(\varphi(\rho(\mathbf{u}))) \in$ $\Delta_{\lambda}\left(\varphi(\rho(\mathbf{u})) \subseteq \Delta_{\lambda}(\varphi(H)) \subseteq \Delta_{\lambda}(A)\right.$, whence $f\left(\rho^{-1}(H)\right) \subseteq \Delta_{\lambda}(A)$.

Finally, we show that $f\left(\{0,1\}^{\mathbb{N}} \backslash \rho^{-1}(H)\right) \subseteq \Delta_{\lambda}(\mathbb{R} \backslash A)$. Namely, if $\mathbf{u} \in\{0,1\}^{\mathbb{N}} \backslash \rho^{-1}(H)=\rho^{-1}\left(\{0,1\}^{\mathbb{N}} \backslash\right.$ $H)$, then $f(\mathbf{u})=\mathcal{S}(\varphi(\rho(\mathbf{u}))) \in \Delta_{\lambda}\left(\varphi(\rho(\mathbf{u})) \subseteq \Delta_{\lambda}\left(\varphi\left(\{0,1\}^{\mathbb{N}} \backslash H\right)\right) \subseteq \Delta_{\lambda}\left(I_{\lambda} \backslash A\right) \subseteq \Delta_{\lambda}(\mathbb{R} \backslash A)\right.$, whence $f\left(\{0,1\}^{\mathbb{N}} \backslash \rho^{-1}(H)\right) \subseteq \Delta_{\lambda}(\mathbb{R} \backslash A)$.

We can now prove Theorem 2.3. For the benefit of the reader we first recall the statement of Theorem 2.3 . 
Theorem 2.3. Lower bounds for arbitrary measures: completeness. Let $\lambda$ be a Borel measure on $\mathbb{R}^{d}$ and assume that $\lambda$ is aspherical, that $\lambda$ has a continuous selector, and that $\lambda$ is admissible. Let $\gamma$ be an ordinal with $\gamma<\omega_{1}$.

(1) If $A \subseteq I_{\lambda}$ is $\Pi_{\gamma}^{0}\left(I_{\lambda}\right)$-complete, then $\Delta_{\lambda}(A)$ is $\begin{cases}\Pi_{\gamma+1}^{0} \text {-complete } & \text { for } 3 \leq \gamma<\omega ; \\ \Pi_{\gamma}^{0} \text {-complete } & \text { for } \omega \leq \gamma .\end{cases}$

(2) If $A \subseteq I_{\lambda}$ is $\Sigma_{\gamma}^{0}\left(I_{\lambda}\right)$-complete, then $\Delta_{\lambda}(A)$ is $\begin{cases}\Sigma_{\gamma+1}^{0} \text {-complete } & \text { for } 3 \leq \gamma<\omega ; \\ \Sigma_{\gamma}^{0} \text {-complete } & \text { for } \omega \leq \gamma .\end{cases}$

Proof.

(1) We divide the proof into the following two parts.

Part 1: Assume that $3 \leq \gamma<\omega$. For convenience write $n=\gamma \in \mathbb{N}$. Also, let $\rho:\{0,1\}^{\mathbb{N}} \rightarrow\{0,1\}^{\mathbb{N}}$ be the map in (6.8), and let $H_{1}, H_{2}, \ldots$ be the sets in (6.9). We must now prove that $\Delta_{n+1}(A)$ is $\Pi_{n+1}^{0}$-hard and that $\Delta_{n+1}(A)$ in $\Pi_{n+1}^{0}$.

We first prove that $\Delta_{n+1}(A)$ is $\Pi_{n+1}^{0}$-hard. Indeed, since $A$ is $\Pi_{n}^{0}\left(I_{\lambda}\right)$-hard and $H_{n} \in \Pi_{n}^{0}$, we conclude that

$$
\left(\{0,1\}^{\mathbb{N}} ; H_{n}\right) \underset{\mathrm{w}}{\leq}\left(I_{\lambda} ; A\right),
$$

and it therefore follows from Proposition 6.4 that

$$
\left(\{0,1\}^{\mathbb{N}} ; H_{n+1}\right)=\left(\{0,1\}^{\mathbb{N}} ; \rho^{-1}\left(H_{n}\right)\right) \underset{\mathrm{w}}{\leq}\left(\mathbb{R} ; \Delta_{\lambda}(A)\right) .
$$

Finally, since $H_{n+1}$ is $\Pi_{n+1}^{0}$-hard it now follows from $(6.10)$ that $\Delta_{n+1}(A)$ is $\Pi_{n+1}^{0}$-hard.

Next, we prove that $\Delta_{\lambda}(A)$ in $\Pi_{n+1}^{0}$. Since $A \in \Pi_{n}^{0}\left(I_{\lambda}\right)$, we conclude that there is a set $E \in \Pi_{n}^{0}(\mathbb{R})$ such that $A=E \cap I_{\lambda}$. Also, since $\Delta_{\lambda}(M)=\varnothing$ for all $M \subseteq \mathbb{R} \backslash I_{\lambda}$, we conclude that

$$
\Delta_{\lambda}(E)=\Delta_{\lambda}\left(E \cap I_{\lambda}\right) .
$$

Furthermore, because $E \in \Pi_{n}^{0}(\mathbb{R})$, it follows from Theorem 2.1 that

$$
\Delta_{\lambda}(E) \in \Pi_{n+1}^{0}
$$

Finally, combining (6.11) and (6.12) and using the fact that $A=E \cap I_{\lambda}$, we conclude that $\Delta_{\lambda}(A)=$ $\Delta_{\lambda}\left(E \cap I_{\lambda}\right)=\Delta_{\lambda}(E) \in \Pi_{n+1}^{0}$.

Part 2: Assume that $\omega \leq \gamma$. We must now prove that $\Delta_{\lambda}(A)$ is $\Pi_{\gamma}^{0}$-hard and that $\Delta_{\lambda}(A)$ in $\Pi_{\gamma}^{0}$.

We first prove that $\Delta_{\lambda}(A)$ is $\Pi_{\gamma}^{0}$-hard. Indeed, since $A$ is $\Pi_{\gamma}^{0}\left(I_{\lambda}\right)$-hard, we conclude that $A$ is $\Pi_{\gamma}^{0}(\mathbb{R})$ hard, and it therefore follows from Theorem 2.2 that $\Delta_{\lambda}(A)$ in $\Pi_{\gamma}^{0}$-hard.

Next, we prove that $\Delta_{\lambda}(A)$ in $\Pi_{\gamma}^{0}$. Since $A \in \Pi_{\gamma}^{0}\left(I_{\lambda}\right)$, we conclude that there is $E \in \Pi_{\gamma}^{0}(\mathbb{R})$ such that $A=E \cap I_{\lambda}$. Using an argument similar to the proof of (6.11) we conclude that

$$
\Delta_{\lambda}(E)=\Delta_{\lambda}\left(E \cap I_{\lambda}\right)
$$

Furthermore, because $E \in \Pi_{\gamma}^{0}(\mathbb{R})$, it follows from Theorem 2.1 that

$$
\Delta_{\lambda}(E) \in \Pi_{\gamma}^{0}
$$

Finally, combining (6.13) and (6.14) and using the fact that $A=E \cap I_{\lambda}$, we conclude that $\Delta_{\lambda}(A)=$ $\Delta_{\lambda}\left(E \cap I_{\lambda}\right)=\Delta_{\lambda}(E) \in \Pi_{\gamma}^{0}$.

(2) The proof of this statement is similar to the proof of the statement in Part (1) and is therefore omitted. 


\section{Proofs of Theorem 3.4 and Theorem 3.6.}

We start be introducing some notation that will be used throughout Section 7 . For a set $I$ and a positive integer $n$, write

$$
\begin{aligned}
I^{n} & =\left\{i_{i} \ldots i_{n} \mid i_{j} \in \Lambda\right\}, \\
I^{*} & =\bigcup_{k} I^{k},
\end{aligned}
$$

i.e. $I^{n}$ is the set of all finite strings $\mathbf{i}=i_{1} \ldots i_{n}$ of length $n$ with $i_{j} \in I$, and $I^{*}$ is the set of all finite strings $\mathbf{i}=i_{1} \ldots i_{n}$ with $i_{j} \in I$. Also, write

$$
I^{\mathbb{N}}=\left\{i_{i} i_{2} \ldots \mid i_{j} \in I\right\}
$$

i.e. $I^{\mathbb{N}}$ is the set of all infinite strings $\mathbf{i}=i_{1} i_{2} \ldots$ with $i_{j} \in I$. If $\mathbf{i}=i_{1} i_{2} \ldots \in I^{\mathbb{N}}$ and $n$ is a positive integer, then we will write $\mathbf{i} \mid n=i_{1} \ldots i_{n}$ for the truncation of $\mathbf{i}$ to the $n$ 'th place. Also, if $\mathbf{i}=i_{1} \ldots i_{n} \in I^{*}$ and $\mathbf{j}=j_{1} \ldots j_{m} \in I^{*}$, then we write $\mathbf{i j}=i_{1} \ldots i_{n} j_{1} \ldots j_{m} \in I^{*}$ for the concatenation of $\mathbf{i}$ and $\mathbf{j}$; similarly, if $\mathbf{i}=i_{1} \ldots i_{n} \in I^{*}$ and $\mathbf{j}=j_{1} j_{2} \ldots \in I^{\mathbb{N}}$, then we write $\mathbf{i j}=i_{1} \ldots i_{n} j_{1} j_{2} \ldots \in I^{\mathbb{N}}$ for the concatenation of $\mathbf{i}$ and $\mathbf{j}$. In addition, if $\mathbf{i}=i_{1} \ldots i_{n} \in I^{*}$, then we write $|\mathbf{i}|=n$ for the length of $\mathbf{i}$.

Next, we introduce the following notation for frequencies, namely, fix $i \in I$ and a positive integer $n$. For $\mathbf{i}=i_{1} i_{2} \ldots \in I^{\mathbb{N}}$ and $i \in I$, write

$$
\pi_{i}(\mathbf{i} ; n)=\frac{\left|\left\{k \leq n \mid i_{k}=i\right\}\right|}{n}
$$

i.e. $\pi_{i}(\mathbf{i} ; n)$ is the frequency of the "digit" $i$ amongst the first $n$ "digits" of the string $\mathbf{i}$. We also write

$$
\boldsymbol{\pi}(\mathbf{i} ; n)=\left(\pi_{i}(\mathbf{i} ; n)\right)_{i \in I},
$$

i.e. $\boldsymbol{\pi}(\mathbf{i} ; n)$ is the vector of frequencies of the "digits" amongst the first $n$ "digits" of the string $\mathbf{i}$. Also, for a finite string $\mathbf{i}=i_{1} \ldots i_{n} \in I^{*}$ and $i \in I$, write

$$
\pi_{i}(\mathbf{i})=\frac{\left|\left\{k \leq|\mathbf{i}| \mid i_{k}=i\right\}\right|}{|\mathbf{i}|}
$$

i.e. $\pi_{i}(\mathbf{i})$ is the frequency of the "digit" $i$ amongst the "digits" of the finite string $\mathbf{i}$.

Finally, we introduce some notation related to self-similar measures. Let $S_{i}: \mathbb{R}^{d} \rightarrow \mathbb{R}^{d}$ for $i=$ $1, \ldots, N$ be contracting similarities and let $\left(p_{1}, \ldots, p_{N}\right)$ be a probability vector. For each $i$, we denote the Lipschitz constant of $S_{i}$ by $r_{i} \in(0,1)$. Let $K$ and $\mu$ be the self-similar set and the self-similar measure associated with the list $\left(S_{1}, \ldots, S_{N}, p_{1}, \ldots, p_{N}\right)$, i.e. $K$ is the unique non-empty compact subset of $\mathbb{R}^{d}$ satisfying (3.1), i.e.

$$
K=\bigcup_{i} S_{i}(K)
$$

and $\mu$ is the unique Borel probability measure on $\mathbb{R}^{d}$ satisfying (3.2), i.e.

$$
\mu=\sum_{i} p_{i} \mu \circ S_{i}^{-1}
$$

Write

and for $\mathbf{i}=i_{1} \ldots i_{n} \in \Sigma^{*}$, let

$$
\Sigma=\{1, \ldots, N\},
$$

$$
r_{\mathbf{i}}=r_{i_{1}} \cdots r_{i_{n}}, \quad p_{\mathbf{i}}=p_{i_{1}} \cdots p_{i_{n}}, \quad S_{\mathbf{i}}=S_{i_{1}} \cdots S_{i_{n}} .
$$

Also, recall that we write

$$
\alpha_{\min }=\min _{i=1, \ldots, N} \frac{\log p_{i}}{\log r_{i}}, \quad \alpha_{\max }=\max _{i=1, \ldots, N} \frac{\log p_{i}}{\log r_{i}} .
$$

Finally, we define the map $\pi: \Sigma^{\mathbb{N}} \rightarrow \mathbb{R}^{d}$ by

$$
\pi(\mathbf{i})=\bigcap_{n} S_{\mathbf{i} \mid n}(K) .
$$

If the SSC is satisfied, then it is well-known that $\pi$ is a bi-Lipschitz map between $\Sigma^{\mathbb{N}}$ and $K$. Proposition 7.1 below is well-known and shows that the multifractal decomposition sets $\Delta_{\mu}(\alpha)$ of the self-similar measure $\mu$ can be expressed "symbolically" using the function $\pi$; the "symbolic" representation of $\Delta_{\mu}(\alpha)$ in Proposition 7.1 plays an important role in the proofs of Theorem 3.4 and Theorem 3.6. 
Proposition 7.1. Let $\mu$ be the self-similar measure in (3.2) and assume that the SSC is satisfied.

(1) We have $I_{\mu}=\left[\alpha_{\min }, \alpha_{\max }\right]$.

(2) For $\alpha \in \mathbb{R}$, we have

$$
\pi\left\{\mathbf{i} \in \Sigma^{\mathbb{N}} \mid \lim _{n} \frac{\log p_{\mathbf{i} \mid n}}{\log r_{\mathbf{i} \mid n}}=\alpha\right\}=\left\{\begin{array}{l|l}
x \in \mathbb{R}^{d} \mid \lim _{r \searrow 0} \frac{\log \mu(B(x, r))}{\log r}=\alpha
\end{array} .\right.
$$

(3) We have

$$
\pi\left\{\begin{array}{l|l}
\mathbf{i} \in \Sigma^{\mathbb{N}} \mid \frac{\log p_{\mathbf{i} \mid n}}{\log r_{\mathbf{i} \mid n}} \text { diverges as } n \rightarrow \infty
\end{array}\right\}=\left\{x \in \mathbb{R}^{d} \mid \begin{array}{l}
\frac{\log \mu(B(x, r))}{\log r} \text { diverges as } r \searrow 0
\end{array}\right\} .
$$

Proof.

This is well-known, see, for example, $[\mathrm{Fa}]$ or $[\mathrm{Pe}]$

We now turn towards the proofs of Theorem 3.4 and Theorem 3.6. The proofs are based on two Wadge reductions. The outline of the proof is the following:

- In Section 7.1 we construct a useful auxiliary function $F$; the function $F$ plays a major role in the constructing of the Wadge reductions needed to prove Theorem 3.4 and Theorem 3.6. We also apply the function $F$ to prove Lemma 3.1 .

- The first Wadge reduction is given in Section 7.2 (Proposition 7.4) .

- The second reduction is given in Section 7.3 (Proposition 7.6).

- Finally, in Sections 7.4-7.5 we apply the reductions from Sections 7.2-7.3 to prove Theorem 3.4 and Theorem 3.6.

7.1. The map $F$ and the proof of Lemma 3.1. In this section we construct the auxiliary map $F$. The function $F$ plays a major role in the constructing of the Wadge reductions needed to prove Theorem 3.4 and Theorem 3.6. The function $F$ will also be applied to prove Lemma 3.1.

Proposition 7.2. Let $I$ be a finite set and let $\left(a_{\mathbf{i}}\right)_{\mathbf{i} \in I^{*}}$ be a family of real numbers. Write

$$
a_{\min }=\min _{i \in I} a_{i}, \quad a_{\max }=\max _{i \in I} a_{i}
$$

and choose $i_{\min }, i_{\max } \in I$ such that

$$
a_{i_{\min }}=a_{\min }, \quad a_{i_{\max }}=a_{\max } .
$$

Assume that

(i) For all $\mathbf{i} \in I^{*}$, we have $a_{\mathbf{i} i_{\min }} \leq a_{\mathbf{i}} \leq a_{\mathbf{i} i_{\max }}$.

(ii) There is a constant $c>0$ such that for all $\mathbf{i} \in \Sigma^{*}$ and all $i \in I$, we have $\left|a_{\mathbf{i} i}-a_{\mathbf{i}}\right| \leq \frac{c}{|\mathbf{i}|}$.

(iii) For all $\mathbf{i} \in I^{*}$, we have $a_{\mathbf{i} i_{\min }^{n}} \rightarrow a_{\min }$ and $a_{\mathbf{i} i_{\max }^{n}} \rightarrow a_{\max }$

Then there is a continuous function $F:\left(a_{\min }, a_{\max }\right)^{\mathbb{N}} \rightarrow I^{\mathbb{N}}$ and a string $\mathbf{k} \in I^{*}$ such that

$$
\begin{array}{ll}
F(t t \ldots) \rightarrow \mathbf{k} i_{\min } i_{\min } \ldots & \text { as } t \searrow a_{\min } \text { with } t \in\left(a_{\min }, a_{\max }\right), \\
F(t t \ldots) \rightarrow \mathbf{k} i_{\max } i_{\max } \ldots & \text { as } t \nearrow a_{\max } \text { with } t \in\left(a_{\min }, a_{\max }\right),
\end{array}
$$

and for all $\mathbf{t}=t_{1} t_{2} \ldots \in\left(a_{\min }, a_{\max }\right)^{\mathbb{N}}$, we have

$$
\begin{aligned}
& \liminf _{n} a_{F(\mathbf{t}) \mid n}=\liminf _{n} t_{n}, \\
& \limsup _{n} a_{F(\mathbf{t}) \mid n}=\limsup _{n} t_{n} .
\end{aligned}
$$

Proof.

Let $\mathbf{t}=t_{1} t_{2} \ldots \in\left(a_{\min }, a_{\max }\right)^{\mathbb{N}}$. We define a sequence $\left(\mathbf{i}_{n}(\mathbf{t})\right)_{n}$ of strings $\mathbf{i}_{n}(\mathbf{t}) \in I^{*}$ inductively such that for all $n$, we have:

(1) $a_{\mathbf{i}_{1}(\mathbf{t}) \ldots \mathbf{i}_{n}(\mathbf{t}) \mathbf{i}_{n+1}(\mathbf{t}) \mid k}$ is a monotone function of $k$ for $\left|\mathbf{i}_{1}(\mathbf{t}) \ldots \mathbf{i}_{n}(\mathbf{t})\right| \leq k \leq\left|\mathbf{i}_{1}(\mathbf{t}) \ldots \mathbf{i}_{n}(\mathbf{t}) \mathbf{i}_{n+1}(\mathbf{t})\right|$;

(2) $\left|a_{\mathbf{i}_{1}(\mathbf{t}) \ldots \mathbf{i}_{n}(\mathbf{t}) \mathbf{i}_{n+1}(\mathbf{t})}-t_{n}\right| \leq \frac{c}{n}$. 
Below we construct the sequence $\left(\mathbf{i}_{n}(\mathbf{t})\right)_{n}$ inductively.

The start of the induction.

Choose any $\mathbf{k} \in I^{*}$ and put $\mathbf{i}_{1}(\mathbf{t})=\mathbf{k}$. Note that Conditions (1)-(2) are satisfied. This completes the start of the induction.

The inductive step.

Assume that the strings $\mathbf{i}_{1}(\mathbf{t}), \ldots, \mathbf{i}_{n}(\mathbf{t}) \in I^{*}$ have been chosen such that Conditions (1)-(2) are satisfied. We will now construct the string $\mathbf{i}_{n+1}(\mathbf{t})$.

If $a_{\mathbf{i}_{1}(\mathbf{t}) \ldots \mathbf{i}_{n}(\mathbf{t})}<t_{n}$, then the fact that $t_{n} \in\left(a_{\min }, a_{\max }\right)$ combined with the fact that the sequence $a_{\mathbf{i}_{1}(\mathbf{t}) \ldots \mathbf{i}_{n}(\mathbf{t}) i_{\max }^{k}}$ is increasing (by Condition (i)) with $\lim _{k} a_{\mathbf{i}_{1}(\mathbf{t}) \ldots \mathbf{i}_{n}(\mathbf{t}) i_{\max }^{k}}=a_{\max }$ (by Condition (iii)), imply that we can choose a unique positive $m_{n+1}(\mathbf{t})$ such that the following is satisfied: if we put $\mathbf{i}_{n+1}(\mathbf{t})=i_{\max }^{m_{n+1}(\mathbf{t})}$ and write $\mathbf{i}_{n+1}^{-}(\mathbf{t})=i_{\max }^{m_{n+1}(\mathbf{t})-1}$, then

$$
a_{\mathbf{i}_{1}(\mathbf{t}) \ldots \mathbf{i}_{n}(\mathbf{t}) \mathbf{i}_{n+1}^{-}(\mathbf{t})}<t_{n} \leq a_{\mathbf{i}_{1}(\mathbf{t}) \ldots \mathbf{i}_{n}(\mathbf{t}) \mathbf{i}_{n+1}(\mathbf{t})} .
$$

If $t_{n} \leq a_{\mathbf{i}_{1}(\mathbf{t}) \ldots \mathbf{i}_{n}(\mathbf{t})}$, then the fact that $t_{n} \in\left(a_{\min }, a_{\max }\right)$ combined with the fact that the sequence $a_{\mathbf{i}_{1}(\mathbf{t}) \ldots \mathbf{i}_{n}(\mathbf{t}) i_{\min }^{k}}$ is decreasing (by Condition (i)) with $\lim _{k} a_{\mathbf{i}_{1}(\mathbf{t}) \ldots \mathbf{i}_{n}(\mathbf{t}) i_{\min }^{k}}=a_{\min }$ (by Condition (iii)), imply that we can choose a unique positive $m_{n+1}(\mathbf{t})$ such that the following is satisfied: if we put $\mathbf{i}_{n+1}(\mathbf{t})=i_{\min }^{m_{n+1}(\mathbf{t})}$ and write $\mathbf{i}_{n+1}^{-}(\mathbf{t})=i_{\min }^{m_{n+1}(\mathbf{t})-1}$, then

$$
a_{\mathbf{i}_{1}(\mathbf{t}) \ldots \mathbf{i}_{n}(\mathbf{t}) \mathbf{i}_{n+1}(\mathbf{t})}<t_{n} \leq a_{\mathbf{i}_{1}(\mathbf{t}) \ldots \mathbf{i}_{n}(\mathbf{t}) \mathbf{i}_{n+1}^{-}(\mathbf{t})} .
$$

Next, we show that Conditions (1)-(2) are satisfied. Indeed, it follows from the construction of $\mathbf{i}_{n+1}(\mathbf{t})$ and Condition (i) that Condition (1) is satisfied. It also follows from (7.7) and (7.8) and Condition (ii) that $\left|a_{\mathbf{i}_{1}(\mathbf{t}) \ldots \mathbf{i}_{n}(\mathbf{t}) \mathbf{i}_{n+1}(\mathbf{t})}-t_{n}\right| \leq\left|a_{\mathbf{i}_{1}(\mathbf{t}) \ldots \mathbf{i}_{n}(\mathbf{t}) \mathbf{i}_{n+1}(\mathbf{t})}-a_{\mathbf{i}_{1}(\mathbf{t}) \ldots \mathbf{i}_{n}(\mathbf{t}) \mathbf{i}_{n+1}^{-}(\mathbf{t})}\right| \leq \frac{c}{\left|\mathbf{i}_{1}(\mathbf{t}) \ldots \mathbf{i}_{n}(\mathbf{t}) \mathbf{i}_{n+1}^{-}(\mathbf{t})\right|} \leq \frac{c}{n}$ (because $\left.\left|\mathbf{i}_{1}(\mathbf{t}) \ldots \mathbf{i}_{n}(\mathbf{t}) \mathbf{i}_{n+1}^{-}(\mathbf{t})\right| \geq\left|\mathbf{i}_{1}(\mathbf{t})\right|+\cdots+\left|\mathbf{i}_{n}(\mathbf{t})\right| \geq n\right)$, and Condition (2) is therefore satisfied. This completes the inductive step.

We now define the function $F$ by

$$
F(\mathbf{t})=\mathbf{i}_{1}(\mathbf{t}) \mathbf{i}_{2}(\mathbf{t}) \ldots
$$

Below we show that $F$ is continuous and that $F$ satisfies (7.5) and (7.6).

We first prove that $F$ is continuous. Indeed, since the family $\left(a_{\mathbf{i}}\right)_{\mathbf{i} \in I^{k}}$ is finite for all $k$, for each positive integer $n$ there is an integer $N_{n}$ such that if $\mathbf{t}=t_{1} t_{2} \ldots, \mathbf{u}=u_{1} u_{2} \ldots \in\left(\alpha_{\min }, \alpha_{\max }\right)^{\mathbb{N}}$ with $t_{i}=u_{i}$ for all $i \leq N_{n}$, then $\mathbf{i}_{i}(\mathbf{t})=\mathbf{i}_{i}(\mathbf{u})$ for all $i \leq n$. This clearly implies that $F$ is continuos.

Next, we note that it follows from the construction of $F$ that $F(t t \ldots) \rightarrow \mathbf{k} i_{\min } i_{\min } \ldots$ as $t \searrow a_{\min }$ and $F(t t \ldots) \rightarrow \mathbf{k} i_{\max } i_{\max } \ldots$ as $t \nearrow a_{\max }$ with $t \in\left(a_{\min }, a_{\max }\right)$.

Finally, we note that it follows from Conditions (1)-(2) that $\liminf _{n} a_{F(\mathbf{t}) \mid n}=\liminf _{n} t_{n}$ and $\lim \sup _{n} a_{F(\mathbf{t}) \mid n}=\limsup _{n} t_{n}$ for all $\mathbf{t}=t_{1} t_{2} \ldots \in\left(a_{\min }, a_{\max }\right)^{\mathbb{N}}$. 
We will now apply the function $F$ to prove Lemma 3.1. We first prove the following auxiliary result.

Proposition 7.3. There is a continuous function $S:\left[\alpha_{\min }, \alpha_{\max }\right] \rightarrow \Sigma^{\mathbb{N}}$ such that

$$
S(\alpha) \in\left\{\mathbf{i} \in \Sigma^{\mathbb{N}} \mid \lim _{n} \frac{\log p_{\mathbf{i} \mid n}}{\log r_{\mathbf{i} \mid n}}=\alpha\right\}
$$

for all $\alpha \in\left[\alpha_{\min }, \alpha_{\max }\right]$.

Proof.

For $\mathbf{i} \in \Sigma^{\mathbb{N}}$, let $a_{\mathbf{i}}=\frac{\log p_{\mathbf{i}}}{\log r_{\mathbf{i}}}$, and choose $i_{\min }, i_{\max } \in\{1, \ldots, N\}$ such that $a_{i_{\min }}=\frac{\log p_{i_{\min }}}{\log r_{i_{\min }}}=\alpha_{\min }$ and $a_{i_{\max }}=\frac{\log p_{i_{\max }}}{\log r_{i_{\min }}}=\alpha_{\max }$. It is easily seen that the family $\left(a_{\mathbf{i}}\right)_{\mathbf{i} \in \Sigma^{\mathbb{N}}}$ satisfies Conditions (i)-(iii) in Proposition 7.2, and we therefore conclude from Proposition 7.2 that there is a continuous function $F:\left(\alpha_{\min }, \alpha_{\max }\right)^{\mathbb{N}} \rightarrow \Sigma^{\mathbb{N}}$ and a string $\mathbf{k} \in \Sigma^{*}$ such that $F(t t \ldots) \rightarrow \mathbf{k} i_{\min } i_{\min } \ldots$ as $t \searrow a_{\min }$ and $F(t t \ldots) \rightarrow \mathbf{k} i_{\max } i_{\max } \ldots$ as $t \nearrow a_{\max }$ with $t \in\left(a_{\min }, a_{\max }\right)$, and $\liminf _{n} a_{F(\mathbf{t}) \mid n}=\liminf _{n} t_{n}$ and $\lim \sup _{n} a_{F(\mathbf{t}) \mid n}=\limsup _{n} t_{n}$ for all $\mathbf{t}=t_{1} t_{2} \ldots \in\left(\alpha_{\min }, \alpha_{\max }\right)^{\mathbb{N}}$. Now define $S:\left[\alpha_{\min }, \alpha_{\max }\right] \rightarrow \Sigma^{\mathbb{N}}$ by

$$
S(\alpha)= \begin{cases}\mathbf{k} i_{\min } i_{\min } \ldots & \text { for } \alpha=\alpha_{\min } ; \\ F(\alpha \alpha \ldots) & \text { for } \alpha \in\left(\alpha_{\min }, \alpha_{\max }\right) ; \\ \mathbf{k} i_{\max } i_{\max } \ldots & \text { for } \alpha=\alpha_{\max } .\end{cases}
$$

It is clear that $S$ is continuous. Also, if $\alpha \in\left(\alpha_{\min }, \alpha_{\max }\right)$, then we conclude that $\lim \inf _{n} a_{S(\alpha) \mid n}=$ $\liminf _{n} a_{F(\alpha \alpha \ldots) \mid n}=\liminf _{n} \alpha=\alpha$ and $\operatorname{lim\operatorname {sup}_{n}} a_{S(\alpha) \mid n}=\operatorname{lim\operatorname {sup}_{n}} a_{F(\alpha \alpha \ldots) \mid n}=\limsup _{n} \alpha=\alpha$, whence $\frac{\log p_{S(\alpha) \mid n}}{\log r_{S(\alpha) \mid n}}=a_{S(\alpha) \mid n} \rightarrow \alpha$. Finally, if $\alpha \in\left\{\alpha_{\min }, \alpha_{\max }\right\}$, then clearly $\frac{\log p_{S(\alpha) \mid n}}{\log r_{S(\alpha) \mid n}} \rightarrow \alpha$.

We can now prove Lemma 3.1.

Lemma 3.1. Let $\mu$ be the self-similar measure in (3.2) and assume that the SSC is satisfied. Then the measure $\mu$ is aspherical, the measure $\mu$ has a continuous selector, and the measure $\mu$ is admissible.

Proof.

It follows from $[\mathrm{Ma}]$ that $\mu$ is aspherical.

Next we prove that $\mu$ has a continuous selector. Indeed, it follows from Proposition 7.3 that we can find a continuous function $S:\left[\alpha_{\min }, \alpha_{\max }\right] \rightarrow \Sigma^{\mathbb{N}}$ such that

$$
S(\alpha) \in\left\{\mathbf{i} \in \Sigma^{\mathbb{N}} \mid \lim _{n} \frac{\log p_{\mathbf{i} \mid n}}{\log r_{\mathbf{i} \mid n}}=\alpha\right\}
$$

for all $\alpha \in\left[\alpha_{\min }, \alpha_{\max }\right]$. Let $\pi: \Sigma^{\mathbb{N}} \rightarrow \mathbb{R}^{d}$ be the function in (7.4) and define $\mathcal{S}:\left[\alpha_{\min }, \alpha_{\max }\right] \rightarrow \mathbb{R}^{d}$ by $\mathcal{S}=\pi \circ S$. The $\mathcal{S}$ is continuous and it follows from Proposition 7.2 that

$$
\mathcal{S}(\alpha)=\pi(S(\alpha)) \in \pi\left\{\mathbf{i} \in \Sigma^{\mathbb{N}} \mid \lim _{n} \frac{\log p_{\mathbf{i} \mid n}}{\log r_{\mathbf{i} \mid n}}=\alpha\right\}=\left\{x \in \mathbb{R}^{d} \mid \lim _{r \searrow 0} \frac{\log \mu(B(x, r))}{\log r}=\alpha\right\}=\Delta_{\mu}(\alpha)
$$

for all $\alpha \in\left[\alpha_{\min }, \alpha_{\max }\right]$.

Finally, since $I_{\mu}=\left[\alpha_{\min }, \alpha_{\max }\right]$, we conclude that $\mu$ is admissible.

7.2. The first reduction. The purpose of this section is to prove Proposition 7.4 establishing the first Wadge reduction used in the proofs of Theorem 3.4 and Theorem 3.6. Write

$$
C=\left\{\nu_{1} \nu_{2} \ldots \in \mathbb{N}^{\mathbb{N}} \mid \lim _{n} \nu_{n}=\infty\right\}
$$

Also write

$$
\begin{aligned}
& \Xi_{0}=\left\{\mathbf{u} \in\{0,1\}^{\mathbb{N}} \mid \pi_{1}(\mathbf{u} ; n) \rightarrow 0\right\}, \\
& \Xi=\left\{\mathbf{u} \in\{0,1\}^{\mathbb{N}} \mid \liminf _{n} \pi_{1}(\mathbf{u} ; n)=0, \underset{n}{\left.\limsup \pi_{1}(\mathbf{u} ; n)>0\right\} .}\right.
\end{aligned}
$$


Proposition 7.4. The first reduction. We have

$$
\left(\mathbb{N}^{\mathbb{N}} ; C\right) \leq \underset{\mathrm{w}}{\leq}\left(\{0,1\}^{\mathbb{N}} ; \Xi_{0}, \Xi\right)
$$

Proof.

We must show that there is a continuous function $f: \mathbb{N}^{\mathbb{N}} \rightarrow\{0,1\}^{\mathbb{N}}$ such that $f(C) \subseteq \Xi_{0}$ and $f\left(\mathbb{N}^{\mathbb{N}} \backslash C\right) \subseteq$ $\Xi$. For $\mathbf{i} \in\{0,1\}^{\mathbb{N}}$, let $a_{\mathbf{i}}=\pi_{1}(\mathbf{i})$. It is easily seen that the family $\left(a_{\mathbf{i}}\right)_{\mathbf{i} \in \Sigma^{\mathbb{N}}}$ satisfies Condition (i)-(iii) in Proposition 7.2 with $a_{\min }=0$ and $a_{\max }=1$, and we therefore conclude from Proposition 7.2

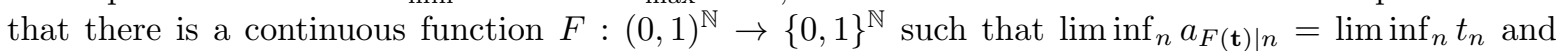
$\limsup _{n} a_{F(\mathbf{t}) \mid n}=\limsup _{n} t_{n}$ for all $\mathbf{t}=t_{1} t_{2} \ldots \in(0,1)^{\mathbb{N}}$. Next, define the map $T: \mathbb{N}^{\mathbb{N}} \rightarrow(0,1)^{\mathbb{N}}$ by $T(\boldsymbol{\nu})=t_{1}(\boldsymbol{\nu}) t_{2}(\boldsymbol{\nu}) \ldots$ for $\boldsymbol{\nu}=\nu_{1} \nu_{2} \ldots \in \mathbb{N}^{\mathbb{N}}$ where

$$
t_{n}(\boldsymbol{\nu})= \begin{cases}\frac{1}{2 n} & \text { for } n \text { odd } \\ \frac{1}{2 \nu_{n}} & \text { for } n \text { even }\end{cases}
$$

(the factor 2 in the denominator in the definition of $t_{n}(\boldsymbol{\nu})$ is included to ensure that $t_{n}(\boldsymbol{\nu}) \in(0,1)$ ). Finally, we define $f: \mathbb{N}^{\mathbb{N}} \rightarrow\{0,1\}^{\mathbb{N}}$ by $f=F \circ T$.

Since $F$ and $T$ are continuous, we conclude that $f$ is continuous.

Next, we show that $f(C) \subseteq \Xi_{0}$. Indeed, if $\boldsymbol{\nu} \in C$, then $t_{n}(\boldsymbol{\nu}) \rightarrow 0$, and so $\liminf _{n} a_{f(\boldsymbol{\nu}) \mid n}=$ $\liminf { }_{n} a_{F(T(\boldsymbol{\nu})) \mid n}=\liminf _{n} t_{n}(\boldsymbol{\nu})=0$ and $\lim \sup _{n} a_{f(\boldsymbol{\nu}) \mid n}=\lim \sup _{n} a_{F(T(\boldsymbol{\nu})) \mid n}=\limsup _{n} t_{n}(\boldsymbol{\nu})=0$, whence $\pi_{1}(f(\boldsymbol{\nu}) \mid n)=a_{f(\boldsymbol{\nu}) \mid n} \rightarrow 0$, i.e. $f(\boldsymbol{\nu}) \in \Xi_{0}$.

Finally, we show that $f\left(\mathbb{N}^{\mathbb{N}} \backslash C\right) \subseteq \Xi$. Namely, if $\boldsymbol{\nu} \in \mathbb{N}^{\mathbb{N}} \backslash C$, then $\liminf _{n} t_{n}(\boldsymbol{\nu})=0$ and

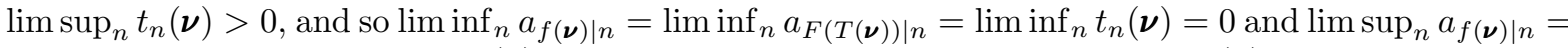
$\limsup _{n} a_{F(T(\boldsymbol{\nu})) \mid n}=\lim \sup _{n} t_{n}(\boldsymbol{\nu})>0$. It follows immediately from this that $f(\boldsymbol{\nu}) \in \Xi$.

7.3. The second reduction. The purpose of this section is to prove Proposition 7.6 establishing the second Wadge reduction used in the proofs of Theorem 3.4 and Theorem 3.6. For $i \in\{1, \ldots, N\}$ and $\mathbf{i}=i_{1} i_{2} \ldots \in \Sigma^{\mathbb{N}}$, let

$$
I_{i, \mathbf{i}}=\left\{k \mid i_{k}=i\right\}
$$

and write

$$
I_{i, \mathbf{i}}=\left\{\sigma_{i, \mathbf{i}}(1), \sigma_{i, \mathbf{i}}(2), \ldots\right\}
$$

where $\sigma_{i, \mathbf{i}}(1)<\sigma_{i, \mathbf{i}}(2)<\ldots$ For $i, j \in\{1, \ldots, N\}$ and $\mathbf{i}=i_{1} i_{2} \ldots \in \Sigma^{\mathbb{N}}$, we now define the function

$$
f_{i, \mathbf{i}, j}:\{0,1\}^{\mathbb{N}} \rightarrow \Sigma^{\mathbb{N}}
$$

as follows. Namely, for $\mathbf{u}=u_{1} u_{2} \ldots \in\{0,1\}^{\mathbb{N}}$, we put

$$
f_{i, \mathbf{i}, j}(\mathbf{u})=j_{1} j_{2} \ldots
$$

where

$$
j_{k}= \begin{cases}i_{k} & \text { for } k \notin I_{i, \mathbf{i}} ; \\ i & \text { for } k \in I_{i, \mathbf{i}} \text { and } k=\sigma_{i, \mathbf{i}}(m) \text { with } u_{m}=0 \\ j & \text { for } k \in I_{i, \mathbf{i}} \text { and } k=\sigma_{i, \mathbf{i}}(m) \text { with } u_{m}=1\end{cases}
$$

Proposition 7.5 below lists the main properties of the function $f_{i, \mathbf{i}, j}$. Recall that we write

$$
\begin{aligned}
\Xi_{\alpha} & =\left\{\mathbf{u} \in\{0,1\}^{\mathbb{N}} \mid \pi_{1}(\mathbf{u} ; n) \rightarrow \alpha\right\}, \\
\Xi & =\left\{\mathbf{u} \in\{0,1\}^{\mathbb{N}} \mid \liminf _{n} \pi_{1}(\mathbf{u} ; n)=0, \underset{n}{\limsup } \pi_{1}(\mathbf{u} ; n)>0\right\} .
\end{aligned}
$$


Proposition 7.5. Fix $\alpha>0$. Let $\mathbf{q}=\left(q_{1}, \ldots, q_{N}\right)$ be a probability vector and let $i, j \in\{1, \ldots, N\}$ and $\mathbf{i} \in \Sigma^{\mathbb{N}}$. Assume that:

(i) $\frac{\sum_{k} q_{k} \log p_{k}}{\sum_{k} q_{k} \log r_{k}}=\alpha$.

(ii) For all real numbers a with $a>0$, we have $\frac{\sum_{k} q_{k} \log p_{k}+a\left(\log p_{i}-\log p_{j}\right)}{\sum_{k} q_{k} \log r_{k}+a\left(\log r_{i}-\log r_{j}\right)} \neq \alpha$.

(iii) $q_{i}>0$.

(iv) $\boldsymbol{\pi}(\mathbf{i} ; n) \rightarrow \mathbf{q}$.

Let $\mathbf{u} \in\{0,1\}^{\mathbb{N}}$ and write $\mathbf{j}=f_{i, \mathbf{i}, j}(\mathbf{u})$. Then the following holds.

(1) The function $f_{i, \mathbf{i}, j}$ s continuous.

(2) If $\pi_{1}(\mathbf{u} ; n) \rightarrow 0$, then $\frac{\log p_{i_{\mid} \mid n}}{\log r_{\mathbf{i} \mid n}} \rightarrow \alpha$.

(3) If $v \geq 0$ and $\left(m_{n}\right)_{n}$ is strictly increasing sequence of integers such that $\pi_{1}\left(\mathbf{u} ; m_{n}\right) \rightarrow v$, then $\frac{\log p_{\mathbf{j} \mid \sigma_{i, \mathbf{i}}\left(m_{n}\right)}}{\log r_{\mathbf{j} \mid \sigma_{i, \mathbf{i}}\left(m_{n}\right)}} \rightarrow \frac{\sum_{k} q_{k} \log p_{k}+q_{i} v\left(\log p_{j}-\log p_{i}\right)}{\sum_{k} q_{k} \log r_{k}+q_{i} v\left(\log r_{j}-\log r_{i}\right)}$

(4) $f_{i, \mathbf{i}, j}\left(\Xi_{0}\right) \subseteq\left\{\mathbf{i} \in \Sigma^{\mathbb{N}} \mid \frac{\log p_{\mathbf{i} \mid n}}{\log r_{\mathbf{i} \mid n}} \rightarrow \alpha\right\}$.

(5) $\quad f_{i, \mathbf{i}, j}(\Xi) \subseteq\left\{\mathbf{i} \in \Sigma^{\mathbb{N}} \mid \frac{\log p_{\mathbf{i} \mid n}}{\log r_{\mathbf{i} \mid n}}\right.$ diverges as $\left.n \rightarrow \infty\right\}$.

Proof.

For each positive integer $n$, write

$$
\nu(\mathbf{u} ; n)=\left|\left\{m \mid \sigma_{i, \mathbf{i}}(m) \leq n, u_{m}=1\right\}\right| .
$$

Next, note that it follows from the definitions of $\nu(\mathbf{u} ; n)$ and $\mathbf{j}=f_{i, \mathbf{i}, j}(\mathbf{u})$ that

$$
\frac{\log p_{\mathbf{j} \mid n}}{\log r_{\mathbf{j} \mid n}}=\frac{\sum_{k} \pi_{k}(\mathbf{i} ; n) \log p_{k}+\frac{\nu(\mathbf{u} ; n)}{n}\left(\log p_{j}-\log p_{i}\right)}{\sum_{k} \pi_{k}(\mathbf{i} ; n) \log r_{k}+\frac{\nu(\mathbf{u} ; n)}{n}\left(\log r_{j}-\log r_{i}\right)} .
$$

We can now prove statements (1)-(5).

(1) This is clear from the construction.

(2) Assume now that $\pi_{1}(\mathbf{u} ; n) \rightarrow 0$. For brevity write $N(\mathbf{u} ; n)=\left|\left\{m \mid \sigma_{i, \mathbf{i}}(m) \leq n\right\}\right|$, and note that $N(\mathbf{u} ; n) \leq n$, whence $\frac{\nu(\mathbf{u} ; n)}{n} \leq \frac{\nu(\mathbf{u} ; n)}{N(\mathbf{u} ; n)}=\pi_{1}(\mathbf{u} ; N(\mathbf{u} ; n)) \rightarrow 0$ (because $\left.\pi_{1}(\mathbf{u} ; n) \rightarrow 0\right)$. Using (7.9), it follows from this and the fact that $\boldsymbol{\pi}(\mathbf{i} ; n) \rightarrow \mathbf{q}\left(\right.$ i.e. $\pi_{k}(\mathbf{i} ; n) \rightarrow q_{k}$ for all $k$ ), that

$$
\begin{aligned}
\frac{\log p_{\mathbf{j} \mid n}}{\log r_{\mathbf{j} \mid n}} & =\frac{\sum_{k} \pi_{k}(\mathbf{i} ; n) \log p_{k}+\frac{\nu(\mathbf{u} ; n)}{n}\left(\log p_{j}-\log p_{i}\right)}{\sum_{k} \pi_{k}(\mathbf{i} ; n) \log r_{k}+\frac{\nu(\mathbf{u} ; n)}{n}\left(\log r_{j}-\log r_{i}\right)} \\
& \rightarrow \frac{\sum_{k} q_{k} \log p_{k}}{\sum_{k} q_{k} \log r_{k}} .
\end{aligned}
$$

Finally, it follows from (i) and assumption (7.10) that $\frac{\log p_{\mathrm{j} \mid n}}{\log r_{\mathrm{j} \mid n}} \rightarrow \alpha$.

(3) Assume now that there is a strictly increasing sequence $\left(m_{n}\right)_{n}$ of positive integers such that $\pi_{1}\left(\mathbf{u} ; m_{n}\right) \rightarrow v$. For brevity write $\sigma(m)=\sigma_{i, \mathbf{i}}(m)$. Noticing that $\nu(\mathbf{u} ; \sigma(m))=m \pi_{1}(\mathbf{u} ; m)$ for all $m$, we conclude from (7.9) that

$$
\begin{aligned}
\frac{\log p_{\mathbf{j} \mid \sigma(m)}}{\log r_{\mathbf{j} \mid \sigma(m)}}= & \frac{\sum_{k} \pi_{k}(\mathbf{i} ; \sigma(m)) \log p_{k}+\frac{\nu(\mathbf{u} ; \sigma(m))}{\sigma(m)}\left(\log p_{j}-\log p_{i}\right)}{\sum_{k} \pi_{k}(\mathbf{i} ; \sigma(m)) \log r_{k}+\frac{\nu(\mathbf{u} ; \sigma(m))}{\sigma(m)}\left(\log r_{j}-\log r_{i}\right)} \\
& =\frac{\sum_{k} \pi_{k}(\mathbf{i} ; \sigma(m)) \log p_{k}+\frac{m}{\sigma(m)} \pi_{1}(\mathbf{u} ; m)\left(\log p_{j}-\log p_{i}\right)}{\sum_{k} \pi_{k}(\mathbf{i} ; \sigma(m)) \log r_{k}+\frac{m}{\sigma(m)} \pi_{1}(\mathbf{u} ; m)\left(\log r_{j}-\log r_{i}\right)} .
\end{aligned}
$$


Next, since clearly $\frac{m}{\sigma(m)}=\pi_{i}(\mathbf{i} ; \sigma(m))$, it follows from (7.11) that

$$
\begin{aligned}
\frac{\log p_{\mathbf{j} \mid \sigma(m)}}{\log r_{\mathbf{j} \mid \sigma(m)}} & =\frac{\sum_{k} \pi_{k}(\mathbf{i} ; \sigma(m)) \log p_{k}+\frac{m}{\sigma(m)} \pi_{1}(\mathbf{u} ; m)\left(\log p_{j}-\log p_{i}\right)}{\sum_{k} \pi_{k}(\mathbf{i} ; \sigma(m)) \log r_{k}+\frac{m}{\sigma(m)} \pi_{1}(\mathbf{u} ; m)\left(\log r_{j}-\log r_{i}\right)} \\
& =\frac{\sum_{k} \pi_{k}(\mathbf{i} ; \sigma(m)) \log p_{k}+\pi_{i}(\mathbf{i} ; \sigma(m)) \pi_{1}(\mathbf{u} ; m)\left(\log p_{j}-\log p_{i}\right)}{\sum_{k} \pi_{k}(\mathbf{i} ; \sigma(m)) \log r_{k}+\pi_{i}(\mathbf{i} ; \sigma(m)) \pi_{1}(\mathbf{u} ; m)\left(\log r_{j}-\log r_{i}\right)} .
\end{aligned}
$$

Finally, since $\pi_{k}\left(\mathbf{i} ; \sigma\left(m_{n}\right)\right) \rightarrow q_{k}$ for all $k$ (because $\boldsymbol{\pi}(\mathbf{i} ; n) \rightarrow \mathbf{q}$ by assumption) and $\pi_{1}\left(\mathbf{u} ; m_{n}\right) \rightarrow v$, we deduce from (7.12) that

$$
\begin{aligned}
\frac{\log p_{\mathbf{j} \mid \sigma\left(m_{n}\right)}}{\log r_{\mathbf{j} \mid \sigma\left(m_{n}\right)}} & =\frac{\sum_{k} \pi_{k}\left(\mathbf{i} ; \sigma\left(m_{n}\right)\right) \log p_{k}+\pi_{i}\left(\mathbf{i} ; \sigma\left(m_{n}\right)\right) \pi_{1}\left(\mathbf{u} ; m_{n}\right)\left(\log p_{j}-\log p_{i}\right)}{\sum_{k} \pi_{k}\left(\mathbf{i} ; \sigma\left(m_{n}\right)\right) \log r_{k}+\pi_{i}\left(\mathbf{i} ; \sigma\left(m_{n}\right)\right) \pi_{1}\left(\mathbf{u} ; m_{n}\right)\left(\log r_{j}-\log r_{i}\right)} \\
& \rightarrow \frac{\sum_{k} q_{k} \log p_{k}+q_{i} v\left(\log p_{j}-\log p_{i}\right)}{\sum_{k} q_{k} \log r_{k}+q_{i} v\left(\log r_{j}-\log r_{i}\right)}
\end{aligned}
$$

(4) This statement follows from (2).

(5) Since $\mathbf{u} \in \Xi$, we can find two strictly increasing sequences $\left(m_{n}\right)_{n}$ and $\left(l_{n}\right)_{n}$ of positive integers and a real number $v$ with $v>0$ such that $\pi_{1}\left(\mathbf{u} ; m_{n}\right) \rightarrow v$ and $\pi_{1}\left(\mathbf{u} ; l_{n}\right) \rightarrow 0$. We conclude from this and $(2)$ that $\frac{\log p_{\mathbf{j} \mid \sigma_{i, \mathbf{i}}\left(m_{n}\right)}}{\log r_{\mathbf{j} \mid \sigma_{i, \mathbf{i}}\left(m_{n}\right)}} \rightarrow \frac{\sum_{k} q_{k} \log p_{k}+q_{i} v\left(\log p_{j}-\log p_{i}\right)}{\sum_{k} q_{k} \log r_{k}+q_{i} v\left(\log r_{j}-\log r_{i}\right)}$ and that $\frac{\log p_{\mathbf{j} \mid \sigma_{i, \mathbf{i}}\left(l_{n}\right)}}{\log r_{\mathbf{j} \mid \sigma_{i, \mathbf{i}}\left(l_{n}\right)}} \rightarrow \frac{\sum_{k} q_{k} \log p_{k}+q_{i} 0\left(\log p_{j}-\log p_{i}\right)}{\sum_{k} q_{k} \log r_{k}+q_{i} 0\left(\log r_{j}-\log r_{i}\right)}=$ $\frac{\sum_{k} q_{k} \log p_{k}}{\sum_{k} q_{k} \log r_{k}}=\alpha$. Finally, since $q_{i} v>0$, it follows from (ii) that $\frac{\sum_{k} q_{k} \log p_{k}+q_{i} v\left(\log p_{j}-\log p_{i}\right)}{\sum_{k} q_{k} \log r_{k}+q_{i} v\left(\log r_{j}-\log r_{i}\right)} \neq \alpha$, and we therefore deduce that $\lim _{n} \frac{\log p_{\mathbf{j} \mid \sigma_{i, \mathbf{i}}\left(k_{n}\right)}}{\log r_{\mathbf{j} \mid \sigma_{i, \mathbf{i}}\left(k_{n}\right)}} \neq \lim _{n} \frac{\log p_{\mathbf{j} \mid \sigma_{i, \mathbf{i}}\left(l_{n}\right)}}{\log r_{\mathbf{j} \mid \sigma_{i, \mathbf{i}}\left(l_{n}\right)}}$. In particular, this shows that the sequence $\left(\frac{\log p_{\mathbf{j} \mid n}}{\log r_{\mathbf{j} \mid n}}\right)_{n}$ diverges.

Proposition 7.6. The second reduction. Let $A \subseteq \mathbb{R}$ and fix $\alpha \in A$. Let $\mathbf{q}=\left(q_{1}, \ldots, q_{N}\right)$ be a probability vector and let $i, j \in\{1, \ldots, N\}$ and $\mathbf{i} \in \Sigma^{\mathbb{N}}$. Assume that:

(i) $\frac{\sum_{k} q_{k} \log p_{k}}{\sum_{k} q_{k} \log r_{k}}=\alpha$.

(ii) For all real numbers a with $a>0$, we have $\frac{\sum_{k} q_{k} \log p_{k}+a\left(\log p_{i}-\log p_{j}\right)}{\sum_{k} q_{k} \log r_{k}+a\left(\log r_{i}-\log r_{j}\right)} \neq \alpha$.

(iii) $q_{i}>0$

(iv) $\boldsymbol{\pi}(\mathbf{i} ; n) \rightarrow \mathbf{q}$.

Then

$$
\left(\{0,1\}^{\mathbb{N}} ; \Xi_{0}, \Xi\right) \underset{\mathrm{w}}{\leq}\left(\mathbb{R} ; \Delta_{\mu}(A), D_{\mu}\right)
$$

Proof.

We must show that there is a continuous function $f:\{0,1\}^{\mathbb{N}} \rightarrow \mathbb{R}^{d}$ such that $f\left(\Xi_{0}\right) \subseteq \Delta_{\mu}(A)$ and $f(\Xi) \subseteq D_{\mu}$. Let $\pi: \Sigma^{\mathbb{N}} \rightarrow \mathbb{R}^{d}$ be the function in (7.4) and define $f:\{0,1\}^{\mathbb{N}} \rightarrow \mathbb{R}^{d}$ by $f=\pi \circ f_{i, \mathbf{i}, j}$. Then $f$ is continuous and it follows from Proposition 7.1 and Proposition 7.5 that

$$
\begin{aligned}
f\left(\Xi_{0}\right) & =\pi\left(f_{i, \mathbf{i}, j}\left(\Xi_{0}\right)\right) \subseteq \pi\left\{\mathbf{i} \in \Sigma^{\mathbb{N}} \mid \frac{\log p_{\mathbf{i} \mid n}}{\log r_{\mathbf{i} \mid n}} \rightarrow \alpha\right\} \\
& =\left\{x \in \mathbb{R}^{d} \mid \lim _{r \searrow 0} \frac{\log \mu(B(x, r))}{\log r}=\alpha\right\} \subseteq\left\{x \in \mathbb{R}^{d} \mid \lim _{r \searrow 0} \frac{\log \mu(B(x, r))}{\log r} \in A\right\}=\Delta_{\mu}(A)
\end{aligned}
$$

and

$$
\begin{aligned}
f(\Xi) & =\pi\left(f_{i, \mathbf{i}, j}(\Xi)\right) \subseteq \pi\left\{\mathbf{i} \in \Sigma^{\mathbb{N}} \mid \frac{\log p_{\mathbf{i} \mid n}}{\log r_{\mathbf{i} \mid n}} \text { diverges as } n \rightarrow \infty\right\} \\
& =\left\{x \in \mathbb{R}^{d} \mid \frac{\log \mu(B(x, r))}{\log r} \text { diverges as } r \searrow 0\right\}=D_{\mu} .
\end{aligned}
$$

This completes the proof 
7.4. Some lemmas. In this section we prove three small auxiliary lemmas.

Lemma 7.7. Let $\left(q_{1}, \ldots, q_{N}\right)$ be a probability vector and write $\frac{\sum_{i} q_{i} \log p_{i}}{\sum_{i} q_{i} \log r_{i}}=\alpha$. Then the following statements are equivalent.

(1) For all $i, j \in\{1, \ldots, N\}$ and all real numbers a with $a>0$ we have $\frac{\sum_{k} q_{k} \log p_{k}+a\left(\log p_{i}-\log p_{j}\right)}{\sum_{k} q_{k} \log r_{k}+a\left(\log r_{i}-\log r_{j}\right)}=$ $\alpha$.

(2) For all $i, j \in\{1, \ldots, N\}$ there is a real number a with $a>0$ such that we have $\frac{\sum_{k} q_{k} \log p_{k}+a\left(\log p_{i}-\log p_{j}\right)}{\sum_{k} q_{k} \log r_{k}+a\left(\log r_{i}-\log r_{j}\right)}=$ $\alpha$.

(3) For all $i \in\{1, \ldots, N\}$, we have $p_{i}=r_{i}^{\alpha}$.

Proof.

$(1) \Rightarrow(2)$ This implication is trivial.

$(2) \Rightarrow(3)$ For brevity write $x=\sum_{k} q_{k} \log p_{k}$ and $y=\sum_{k} q_{k} \log r_{k}$. We first prove the following claim

Claim 1. For all $i, j \in\{1, \ldots, N\}$, we have $\frac{p_{i}}{r_{i}^{\alpha}}=\frac{p_{j}}{r_{j}^{\alpha}}$

Proof of Claim 1. Fix $i, j \in\{1, \ldots, N\}$. It follows from (2) that there is a real number $a$ with $a>0$ such that we have $\frac{\sum_{k} q_{k} \log p_{k}+a\left(\log p_{i}-\log p_{j}\right)}{\sum_{k} q_{k} \log r_{k}+a\left(\log r_{i}-\log r_{j}\right)}=\alpha$, i.e. $\frac{x+a\left(\log p_{i}-\log p_{j}\right)}{y+a\left(\log r_{i}-\log r_{j}\right)}=\frac{x}{y}$. Rearranging this equality and using the fact that $a \neq 0$ gives $y \log \frac{p_{i}}{p_{j}}=x \log \frac{r_{i}}{r_{j}}$, and so $\log \frac{p_{i}}{p_{j}}=\frac{x}{y} \log \frac{r_{i}}{r_{j}}=\alpha \log \frac{r_{i}}{r_{j}}=\log \frac{r_{i}^{\alpha}}{r_{j}^{\alpha}}$. We conclude from this that $\frac{p_{i}}{p_{j}}=\frac{r_{i}^{\alpha}}{r_{j}^{\alpha}}$, i.e. $\frac{p_{i}}{r_{i}^{\alpha}}=\frac{p_{j}}{r_{j}^{\alpha}}$. This completes the proof of Claim 1.

It follows from Claim 1 , that there is a constant $c>0$ such that $\frac{p_{1}}{r_{1}^{\alpha}}=\ldots=\frac{p_{N}}{r_{N}^{\alpha}}=c$, i.e.

$$
p_{i}=c r_{i}^{\alpha} \quad \text { for all } i=1, \ldots, N
$$

Next, we prove that

$$
c=1 \text {. }
$$

Indeed, using the fact that $p_{i}=c r_{i}^{\alpha}$ for all $i=1, \ldots, N$, we conclude that $\alpha=\frac{\sum_{i} q_{i} \log p_{i}}{\sum_{i} q_{i} \log r_{i}}=$ $\frac{\sum_{i} q_{i} \log \left(c r_{i}^{\alpha}\right)}{\sum_{i} q_{i} \log r_{i}}=\frac{\log c}{\sum_{i} q_{i} \log r_{i}}+\alpha$, and so $c=1$.

Finally, it follows from (7.13) and (7.14) that $p_{i}=c r_{i}^{\alpha}=r_{i}^{\alpha}$ for all $i=1, \ldots, N$.

$(3) \Rightarrow(1)$ This implication follows from a simple and direct calculation.

Lemma 7.8. If $\alpha \in\left(\alpha_{\min }, \alpha_{\max }\right)$, then there is a probability vector $\left(q_{1}, \ldots, q_{N}\right)$ with $q_{k}>0$ for all $k$ such that $\frac{\sum_{k} q_{k} \log p_{k}}{\sum_{k} q_{k} \log r_{k}}=\alpha$.

Proof.

Let $\mathbb{P}^{N}$ denote the simplex of $N$ dimensional probability vectors in $\mathbb{R}^{N}$.

Choose $i_{\min }, i_{\max } \in\{1, \ldots, N\}$ such that $\frac{\log p_{i_{\min }}}{\log r_{i_{\min }}}=\alpha_{\min }$ and $\frac{\log p_{i_{\max }}}{\log r_{i_{\max }}}=\alpha_{\max }$, and put $\mathbf{q}_{\min }=$ $\left(\delta_{i, i_{\min }}\right)_{i=1, \ldots, N} \in \mathbb{P}^{N}$ and $\mathbf{q}_{\max }=\left(\delta_{i, i_{\max }}\right)_{i=1, \ldots, N} \in \mathbb{P}^{N}$ (where $\delta_{i, j}$ denotes the Kronecker delta). Next, let $\mathbf{u}=\left(\frac{1}{N}, \ldots, \frac{1}{N}\right)$ denote the uniform $N$ dimensional probability vector in $\mathbb{R}^{N}$ and let $f_{\min }, f_{\max }$ : $[0,1] \rightarrow[0,1]$ be continuous functions with $f_{\min }(0)=1, f_{\min }(1)=0, f_{\max }(0)=0, f_{\max }(1)=1$ and $t(1-t)+f_{\min }(t)+f_{\max }(t)=1$ for all $t \in[0,1]$ (for example, we may put $f_{\min }(t)=(1-t)^{2}$ and $f_{\max }(t)=t$ for $\left.t \in[0,1]\right)$. Finally, for $t \in[0,1]$, define the probability vector $\mathbf{q}_{t}$ by $\mathbf{q}_{t}=t(1-t) \mathbf{u}+$ $f_{\min }(t) \mathbf{q}_{\min }+f_{\max }(t) \mathbf{q}_{\max }$.

Next, define $F: \mathbb{P}^{N} \rightarrow \mathbb{R}$ by $F\left(x_{1}, \ldots, x_{N}\right)=\frac{\sum_{k} x_{k} \log p_{k}}{\sum_{k} x_{k} \log r_{k}}$ and define $f:[0,1] \rightarrow \mathbb{R}$ by $f(t)=F\left(\mathbf{q}_{t}\right)$. The function $f$ is clearly continuous with $f(0)=\alpha_{\min }$ and $f(1)=\alpha_{\max }$, and it therefore follows from the mean value theorem that there is a number $t_{\alpha} \in(0,1)$ such that $f\left(t_{\alpha}\right)=\alpha$. The probability vector $\left(q_{1}, \ldots, q_{N}\right)=\mathbf{q}_{t_{\alpha}}$ is easily seen to have the desired properties. 
Lemma 7.9. Let $K$ be the self-similar set in (3.1) and assume that the SSC is satisfied. Write $s=$ $\operatorname{dim}_{\mathrm{H}}(K)$ and assume that $\left(p_{1}, \ldots, p_{N}\right) \neq\left(r_{1}^{s}, \ldots, r_{N}^{s}\right)$. Fix $\alpha \in\left[\alpha_{\min }, \alpha_{\max }\right]$. Then there is a probability vector $\mathbf{q}=\left(q_{1}, \ldots, q_{N}\right)$ and there are $i, j \in\{1, \ldots, N\}$ and $\mathbf{i} \in \Sigma^{\mathbb{N}}$ such that:

(1) $\frac{\sum_{k} q_{k} \log p_{k}}{\sum_{k} q_{k} \log r_{k}}=\alpha$.

(2) For all real numbers a with $a>0$, we have $\frac{\sum_{k} q_{k} \log p_{k}+a\left(\log p_{i}-\log p_{j}\right)}{\sum_{k} q_{k} \log r_{k}+a\left(\log r_{i}-\log r_{j}\right)} \neq \alpha$.

(3) $q_{i}>0$.

(4) $\boldsymbol{\pi}(\mathbf{i} ; n) \rightarrow \mathbf{q}$.

Proof.

We divide the proof into three cases.

Case 1. Assume that $\alpha \in\left(\alpha_{\min }, \alpha_{\max }\right)$. It follows from Lemma 7.8 that there is a probability vector $\left(q_{1}, \ldots, q_{N}\right)$ with $q_{k}>0$ for all $k$ such that $\frac{\sum_{k} q_{k} \log p_{k}}{\sum_{k} q_{k} \log r_{k}}=\alpha$. Next, we prove that

$$
\left(p_{1}, \ldots, p_{N}\right) \neq\left(r_{1}^{\alpha}, \ldots, r_{N}^{\alpha}\right)
$$

Indeed, otherwise $\left(p_{1}, \ldots, p_{N}\right)=\left(r_{1}^{\alpha}, \ldots, r_{N}^{\alpha}\right)$, whence $\sum_{k} r_{k}^{\alpha}=\sum_{k} p_{k}=1$, and so $\alpha=\operatorname{dim}_{\mathrm{H}}(K)=$ $s$ (see, for example, [Fa]). We therefore conclude that $\left(p_{1}, \ldots, p_{N}\right)=\left(r_{1}^{\alpha}, \ldots, r_{N}^{\alpha}\right)=\left(r_{1}^{s}, \ldots, r_{N}^{s}\right)$. However, this contradicts the assumption that $\left(p_{1}, \ldots, p_{N}\right) \neq\left(r_{1}^{s}, \ldots, r_{N}^{s}\right)$. This completes the proof of (7.15).

Using (7.15), we deduce from Lemma 7.7 that there are indices $i, j \in\{1, \ldots, N\}$, such that for all real numbers $a$ with $a>0$, we have $\frac{\sum_{k} q_{k} \log p_{k}+a\left(\log p_{i}-\log p_{j}\right)}{\sum_{k} q_{k} \log r_{k}+a\left(\log r_{i}-\log r_{j}\right)} \neq \alpha$.

Finally, it is clear that $q_{i}>0$, and that we can choose $\mathbf{i} \in \Sigma^{\mathbb{N}}$ such that $\pi_{k}(\mathbf{i} ; n) \rightarrow q_{k}$ for all $k$.

This completes the proof of the statement in the lemma in Case 1.

Case 2. Assume that $\alpha=\alpha_{\min }$. Choose $i_{\min }, i_{\max } \in\{1, \ldots, N\}$ such that $\frac{\log p_{i_{\min }}}{\log r_{i_{\min }}}=\alpha_{\min }$ and $\frac{\log p_{i_{\max }}}{\log r_{i_{\max }}}=\alpha_{\max }$. Now put $\left(q_{k}\right)_{k}=\left(\delta_{k, i_{\min }}\right)_{k}$, and $i=i_{\max }$ and $j=i_{\max }$.

It is clear that $\frac{\sum_{k} q_{k} \log p_{k}}{\sum_{k} q_{k} \log r_{k}}=\alpha$.

Next, we prove that $\frac{\sum_{k} q_{k} \log p_{k}+a\left(\log p_{i}-\log p_{j}\right)}{\sum_{k} q_{k} \log r_{k}+a\left(\log r_{i}-\log r_{j}\right)} \neq \alpha$ for all real numbers $a$ with $a>0$, i.e. we prove that $\frac{\log p_{i}+a\left(\log p_{i}-\log p_{j}\right)}{\log r_{i}+a\left(\log r_{i}-\log r_{j}\right)} \neq \alpha$ for all real numbers $a$ with $a>0$. Indeed, assume that this is not the case. Hence we can find a real number $a$ with $a>0$ such that $\frac{\log p_{i}+a\left(\log p_{i}-\log p_{j}\right)}{\log r_{i}+a\left(\log r_{i}-\log r_{j}\right)}=\alpha=\frac{\log p_{i}}{\log r_{i}}$. Rearranging this expression gives $a \log \left(r_{i}\right) \log \left(\frac{p_{i}}{p_{j}}\right)=a \log \left(p_{i}\right) \log \left(\frac{r_{i}}{r_{j}}\right)$, and using the fact that $a \neq 0$, this implies that $\log \left(\frac{p_{i}}{p_{j}}\right)=\frac{\log p_{i}}{\log r_{i}} \log \left(\frac{r_{i}}{r_{j}}\right)=\alpha \log \left(\frac{r_{i}}{r_{j}}\right)$, whence $\frac{p_{j}}{r_{j}^{\alpha}}=\frac{p_{i}}{r_{i}^{\alpha}}=1$, and so $\alpha_{\min }=\alpha=\frac{\log p_{j}}{\log r_{j}}=\frac{\log p_{i_{\max }}}{\log r_{i_{\max }}}=\alpha_{\max }$. Now write $t$ for the common value of $\alpha_{\min }$ and $\alpha_{\max }$, i.e. we write $t=\alpha_{\min }=\alpha_{\max }$. Hence, for all $k$, we have $\frac{\log p_{k}}{\log r_{k}} \in\left[\alpha_{\min }, \alpha_{\text {max }}\right]=\{t\}$, whence $p_{k}=r_{k}^{t}$ for all $k$, and so $\sum_{k} r_{k}^{t}=\sum_{k} p_{k}=1$. This implies that $t=\operatorname{dim}_{\mathrm{H}}(K)=s$ (see, for example, $\left.[\mathrm{Fa}]\right)$. We therefore conclude that $\left(p_{1}, \ldots, p_{N}\right)=\left(r_{1}^{t}, \ldots, r_{N}^{t}\right)=$ $\left(r_{1}^{s}, \ldots, r_{N}^{s}\right)$. However, this contradicts the assumption that $\left(p_{1}, \ldots, p_{N}\right) \neq\left(r_{1}^{s}, \ldots, r_{N}^{s}\right)$, and completes the proof.

Finally, it is clear that $q_{i}=1>0$, and that we can choose $\mathbf{i} \in \Sigma^{\mathbb{N}}$ with $\pi_{k}(\mathbf{i} ; n) \rightarrow q_{k}$ for all $k$.

This completes the proof of the statement in the lemma in Case 2.

Case 3. Assume that $\alpha=\alpha_{\max }$. The proof of the statement in Case 3 is similar to the proof in Case 2 and is therefore omitted. 
7.5. The proofs of Theorem 3.4 and Theorem 3.6. Using Proposition 7.4, Proposition 7.6 and Lemma 7.9, will now prove prove Theorem 3.4 and Theorem 3.6. We first prove the following auxiliary result. Recall that we write

$$
C=\left\{\nu_{1} \nu_{2} \ldots \in \mathbb{N}^{\mathbb{N}} \mid \lim _{n} \nu_{n}=\infty\right\}
$$

Theorem 7.10. Let $K$ and $\mu$ be the self-similar set and self-similar measure in (3.1) and (3.2), respectively, and assume that the $S S C$ is satisfied. Write $s=\operatorname{dim}_{\mathrm{H}}(K)$ and assume that $\left(p_{1}, \ldots, p_{N}\right) \neq$ $\left(r_{1}^{s}, \ldots, r_{N}^{s}\right)$. If $A \cap\left[\alpha_{\min }, \alpha_{\max }\right] \neq \varnothing$, then

$$
\left(\mathbb{N}^{\mathbb{N}} ; C\right) \underset{\mathrm{w}}{\leq}\left(\mathbb{R}^{d} ; \Delta_{\mu}(A), D_{\mu}\right)
$$

Proof.

Note that it follows from Proposition 7.4 that

$$
\left(\mathbb{N}^{\mathbb{N}} ; C\right) \underset{\mathrm{w}}{\leq}\left(\{0,1\}^{\mathbb{N}} ; \Xi_{0}, \Xi\right)
$$

Since $A \cap\left[\alpha_{\min }, \alpha_{\max }\right] \neq \varnothing$, we can choose $\alpha \in A \cap\left[\alpha_{\min }, \alpha_{\max }\right]$. It now follows from Lemma 7.9 that there is a probability vector $\mathbf{q}=\left(q_{1}, \ldots, q_{N}\right)$ and that there are $i, j \in\{1, \ldots, N\}$ and $\mathbf{i} \in \Sigma^{\mathbb{N}}$ such that:

$$
\begin{aligned}
& \text { (1) } \frac{\sum_{k} q_{k} \log p_{k}}{\sum_{k} q_{k} \log r_{k}}=\alpha . \\
& \text { (2) For all real numbers } a \text { with } a>0 \text {, we have } \frac{\sum_{k} q_{k} \log p_{k}+a\left(\log p_{i}-\log p_{j}\right)}{\sum_{k} q_{k} \log r_{k}+a\left(\log r_{i}-\log r_{j}\right)} \neq \alpha . \\
& \text { (3) } q_{i}>0 . \\
& \text { (4) } \boldsymbol{\pi}(\mathbf{i} ; n) \rightarrow \mathbf{q} .
\end{aligned}
$$

We therefore conclude from Proposition 7.6 that

$$
\left(\{0,1\}^{\mathbb{N}} ; \Xi_{0}, \Xi\right) \underset{\mathrm{w}}{\leq}\left(\mathbb{R} ; \Delta_{\mu}(A), D_{\mu}\right)
$$

The desired conclusion follows immediately from (7.16) and (7.17).

We can now prove Theorem 3.4 and Theorem 3.6.

Proof of Theorem 3.4.

Since $C$ is $\Pi_{3}^{0}$-complete (see, for example, [Ke, Exercise 23.2]), it follows from Theorem 7.10 that $\Delta_{\mu}(A)$ is $\Pi_{3}^{0}$-hard.

Proof of Theorem 3.6.

Since $C$ is $\Pi_{3}^{0}$-complete (see, for example, [Ke, Exercise 23.2]) and $D_{\mu} \in \Sigma_{3}^{0}$ (by Lemma 3.1 and Lemma $4.3)$, it follows from Theorem 7.10 that $D_{\mu}$ is $\Sigma_{3}^{0}$-complete.

\section{REFERENCES}

BaSc. L. Barreira \& J. Schmeling, Sets of "non-typical" points have full topological entropy and full Hausdorff dimension, Israel J. Math. 116 (2000), 29-70.

Fa. K. J. Falconer, Techniques in Fractal Geometry, John Wiley \& Sons, Ltd., Chichester, 1997.

FeWu. D.-J. Feng \& J. Wu, The Hausdorff dimension of recurrent sets in symbolic spaces, Nonlinearity 14 (2001), $81-85$.

Hu. J. Hutchinson, Fractals and self-similarity, Indiana Univ. Math. J. 30 (1981), 713-747.

Ke. A. Kechris, Classical Descriptive Set Theory, Graduate Texts in Mathematics, Volume 156, Springer Verlag, 1994.

KiLi. H. Ki \& T. Linton, Normal numbers and subsets of $\mathbb{N}$ with given densities, Fundamenta Mathematicae 144 (1994), 163-179.

LoSRa. A. Louveau \& J. Saint Raymond, Borel classes and closed games: Wadge-type and Hurewicz-type results, Trans. AMS 304 (1987), 431-467.

Ma. P. Mattila, On the structure of self-similar fractals, Ann. Acad. Sci. Fenn. Ser. A I Math. 7 (1982), 189-195.

OlWi. L. Olsen \& S. Winter, Normal and non-normal points of self-similar sets and divergence points of self-similar measures, Jour. London Math. Soc. 67 (2003), 103-122.

Pe. Y. Pesin, Dimension Theory in Dynamical Systems. Contemporary Views and Applications, The University of Chicago Press, 1997. 